\title{
Overview of the Cancer Genetics and Pathway Curation tasks of BioNLP Shared Task 2013
}

\author{
Sampo Pyysalo ${ }^{1 *}$, Tomoko Ohta ${ }^{2}$, Rafal Rak ${ }^{3}$, Andrew Rowley ${ }^{3}$, Hong-Woo Chun ${ }^{4}$, Sung-Jae Jung ${ }^{4,5}$, Sung-Pil Choi ${ }^{6}$, \\ Jun'ichi Tsujii', Sophia Ananiadou ${ }^{3}$
}

From BioNLP Shared Task 2013

Sofia, Bulgaria. 9 August 2013

\begin{abstract}
Background: Since their introduction in 2009, the BioNLP Shared Task events have been instrumental in advancing the development of methods and resources for the automatic extraction of information from the biomedical literature. In this paper, we present the Cancer Genetics (CG) and Pathway Curation (PC) tasks, two event extraction tasks introduced in the BioNLP Shared Task 2013. The CG task focuses on cancer, emphasizing the extraction of physiological and pathological processes at various levels of biological organization, and the PC task targets reactions relevant to the development of biomolecular pathway models, defining its extraction targets on the basis of established pathway representations and ontologies.
\end{abstract}

Results: Six groups participated in the CG task and two groups in the PC task, together applying a wide range of extraction approaches including both established state-of-the-art systems and newly introduced extraction methods. The best-performing systems achieved F-scores of 55\% on the CG task and 53\% on the PC task, demonstrating a level of performance comparable to the best results achieved in similar previously proposed tasks.

Conclusions: The results indicate that existing event extraction technology can generalize to meet the novel challenges represented by the CG and PC task settings, suggesting that extraction methods are capable of supporting the construction of knowledge bases on the molecular mechanisms of cancer and the curation of biomolecular pathway models. The CG and PC tasks continue as open challenges for all interested parties, with data, tools and resources available from the shared task homepage.

\section{Background}

The BioNLP Shared Task (BioNLP ST), organized for the third time in 2013, presents open challenges in biomedical natural language processing to all interested parties. The shared task organizers provide task definitions, manually annotated data for method development and evaluation, and tools for the assessment and comparison of information extraction approaches proposed by the community [1-3]. We describe two of the event extraction tasks of the BioNLP ST'13 challenge, the Cancer Genetics (CG) and Pathway Curation (PC) tasks, both of which were newly introduced for the 2013 challenge. This manuscripts

\footnotetext{
* Correspondence: sampo@pyysalo.net

'Department of Information technology, University of Turku, Turku, Finland
} Full list of author information is available at the end of the article extends on the previous papers presenting the tasks at the BioNLP ST workshop $[4,5]$.

Both the CG and PC tasks aim to support knowledge base construction, the over-arching theme of the 2013 BioNLP ST. That is, both tasks are motivated by the need to develop better methods for automatically analyzing the literature at large scale in order to support the creation, maintenance and further development of structured representations of domain knowledge. However, the specific representations and subdomains of biomedical knowledge targeted by the tasks differ substantially, leading to two different information extraction task settings.

The CG task targets the automatic analysis of the literature on cancer, a complex group of genetic diseases that is one of the most common causes of death worldwide. Despite decades of focused research and an ever-increasing 
body of knowledge on its causes and mechanisms, cancer remains imperfectly understood [6,7]. Part of the challenge of building comprehensive knowledge bases on cancer is the sheer volume of information on the topic: for example, a search for "cancer" in the PubMed literature database [8] finds mentions in over three million publications, or in approximately one in every eight papers published in the domain.

By contrast to the disease-oriented focus of the CG task, the goals of the PC task are defined by a specific category of knowledge representation, namely formal models of biomolecular pathways such as the Systems Biology Markup Language (SBML) $[9,10]$ and the Biological Pathway Exchange (BioPAX) language $[11,12]$. Structured models such as these are increasingly applied to capture the best current understanding of complex biomolecular systems and represent it in a way that permits automatic processing [13], and a large number of such models can be found in repositories such as BioModels [14] and PANTHER DB [15]. However, the construction of these models is a demanding task, with large models potentially combining information from thousands of individual publications from among the millions of published papers involving biomolecules and their reactions.

Although cancer knowledge base construction and pathway model curation both face challenges stemming from the enormous size and rapid growth of the biomedical literature, existing tools for the automatic analysis of that literature have not fully addressed the needs of these important tasks. In particular, in biomedical information extraction there has long been considerable focus on the recognition of biomolecular entities and binary relations such as protein-protein interaction involving these entities [16-20], largely to the exclusion of upper levels of biological organization and richer representations of entity relations such as pathway models.

While previous BioNLP ST events have been instrumental in promoting the extraction of more expressive representations in the form of event structures [21,22], they have still been mostly limited to molecular and subcellular level entities and processes (Table 1). As an understanding of cancer requires the ability to associate molecular level causes with cellular, tissue- and organlevel effects and organism-level outcomes, methods following the approaches of these previous shared tasks are only capable of addressing a modest part of the challenge of supporting cancer knowledge base construction. Similarly, although the event structures applied in the BioNLP ST tasks have similar expressivity to pathway models, former tasks in the series have not directly addressed either the specific representation nor the semantics of major pathway formalisms, leaving open significant challenges in mapping between event structures, pathway reactions, and the semantic types of the two [23,24].
Table 1. Properties of selected shared tasks in biomedical information extraction prior to the BioNLP ST 13

\begin{tabular}{lll}
\hline Task & $\begin{array}{l}\text { Levels of biological } \\
\text { organization }\end{array}$ & Representation \\
\hline LLL'05 & Molecular & $\begin{array}{l}\text { Binary relations } \\
\text { Binary relations }\end{array}$ \\
BioCreative II PPI & Molecular & Binary relations \\
BioCreative II.5 & Molecular & \\
IPT & & Event structures \\
BioNLP ST'09 GE & Molecular, subcellular & Binary relations \\
BioCreative III PPI & Molecular & Event structures \\
BioNLP ST'11 GE & Molecular, subcellular & Event structures \\
BioNLP ST'11 EPI & Molecular & Event structures \\
BioNLP ST'11 ID & Molecular, organism & Binary relations \\
BioNLP ST'11 BB & Cellular, anatomical, environment & Event structures \\
BioNLP ST'11 BI & Molecular &
\end{tabular}

Entity mention-focused tasks (detection and normalization) and the supporting tasks of the BioNLP ST are not included.

We believe that the event extraction approach can substantially benefit both cancer knowledge base construction and the curation of biomolecular pathway models, and the BioNLP ST'13 Cancer Genetics and Pathway Curation tasks aim to close the gaps hindering the realization of these benefits. Specifically, the CG task introduces novel extraction targets covering all levels of biological organization ranging from the molecular to the whole organism, further extending the scope of previous tasks in the series through the inclusion of pathological processes and events representing experimenter action. The PC task bases both its representation and its semantics directly on major pathway model standards such as SBML, BioPAX and the Systems Biology Ontology $[25,26]$ to assure the compatibility of extracted information with these efforts. Extensive newly annotated corpora are introduced to support both of these novel extraction tasks.

In the following, we first present the applied event representation and its application in the CG and PC task settings in detail. We next introduce the corpora for the two tasks and the criteria for evaluating the predictions submitted by task participants against their annotation. We then present the task participants and systems, the primary evaluation results and additional analyses of these results, and finally discuss the overall findings of these tasks and present conclusions.

\section{Methods}

\section{Representation}

The BioNLP ST main tasks are information extraction tasks targeting event structures (or events for short) [27] following the general approach first introduced in the BioNLP ST'09 [28]. Events capture information on reactions and processes involving physical entities of interest. In addition to entities and events, the representation 
applied in the tasks involves relations between entities and event modifications that identify additional aspects of extracted events such as speculation and negation. We next present these annotation primitives, and then proceed to present the specific types and their definitions applied in the CG and PC tasks.

\section{Entities}

Each mention of a relevant physical entity is annotated as a contiguous span of text that is assigned a type such as CELL or SIMPLE CHEMICAL from a closed set of entity types defined for the task. Figure 1 shows examples of entity annotation.

The recognition of mentions of physical entities in free text is a very well-studied task both in general-domain and biomedical natural language processing $[29,30]$, and the recognition of many key entity types relevant to the CG and PC tasks has been considered in particular for molecular level entities in a number of tasks in the BioCreative series of community evaluations $[16,17,31]$. Thus, to focus the efforts of participants on the novel aspects of the CG and PC tasks, manually created ("gold standard") physical entity mention annotations are provided to participants also for test data, following a convention first established in the BioNLP ST'09.

\section{Relations}

The CG and PC tasks both define a single relation type, Equiv. Equiv is a symmetric, transitive binary relation that identifies entity mentions as being equivalent in the sense of referring to the same real-world entity. Equiv relations are used to mark local aliases such as abbreviations. Figure 2 shows examples of the relation annotation.

The relation annotations are not an extraction target in the task, and gold standard Equiv annotation is applied also for the test data. The Equiv annotations are used in evaluation when determining if two events match: for event matching, any entities connected by an Equiv relation (directly or transitively) are interchangeable.

\section{Events}

Events, the primary extraction target in BioNLP ST main tasks, are structured annotations, each of which has a type, zero or more participants, and an associated statement in text that expresses the event (the event trigger). As for entity annotations, event types are drawn from a closed, task-specific set, and the event trigger is a contiguous span of characters. Event participants can be either entity or event annotations, the latter allowing

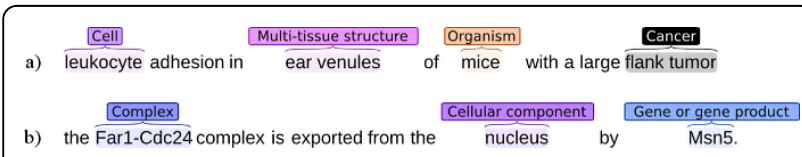

Figure 1 Illustration of entity annotations. a) Cancer Genetics task b) Pathway Curation task. (Illustrations created with BRAT [59])

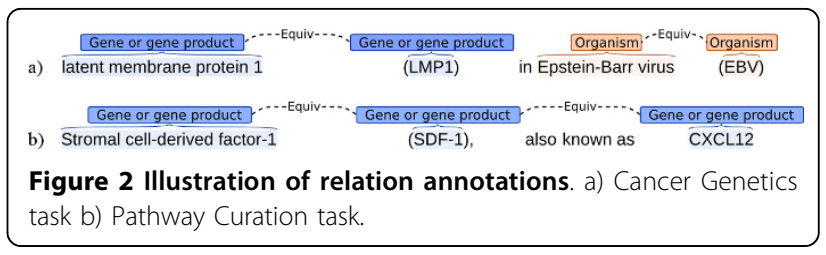

complex event structures where one event is identified as e.g. causing or preventing another. For each event participant, the role that the participant plays in the event is further identified. The number of role types is small and mostly generic, but some moderately taskspecific roles are also defined. The following roles are used in the CG and PC tasks:

Theme Entity or event that undergoes the primary effects of the event. For example, the GENE OR GENE PRODUCT "p53" in "p53 is transcribed" and the DEVELOPMENT event in "regulation of ... development".

Cause Entity or event that is causally active in the event. Example: the "insulin" entity in "insulin regulates VEGF expression" and the NEGATIVE REGULATION event triggered by "inhibition" in "inhibition ... prevented necrosis".

Participant Entity or event that participates in the event in a way that is not specified in detail in the context. Example: GENE OR GENE PRODUCT "TAK1" in "TAK1 is involved in Wnt signaling".

Product Entity that is produced in the event. Example: the COMPLEX "NF-kappa B" in "formation of NF-kappa $B$ ". Not applied in the CG task.

Instrument Entity used to carry out the event. Example: the GENE OR GENE PRODUCT "angiostatin" in "mice were treated with angiostatin". Not applied in the PC task.

Site Part of the Theme entity that is specifically affected by the event. Example: "serine-15" in "phosphorylation of $p 53$ on serine-15"

AtLoc Location where the event takes place. Example: the ORGAN "skin" in "skin tumorigenesis" and the CELLULAR COMPONENT "nucleus" in "Rlm1 resides in the nucleus".

FromLoc Source of movement in events involving change of location. Example: the CELLULAR COMPONENT "mitochondria" in "release of cytochrome c from the mitochondria" and "cytoplasm" in "MAPK translocates from the cytoplasm"

ToLoc Direction or end point of movement in events involving change of location. Example: "lymph node" in "lymph node metastasis" and "plasma membrane" in "RSK1 translocates to the plasma membrane".

Examples of event annotation are shown in Figure 3.

\section{Event modifications}

Both the CG and PC tasks involve the modification types NEGATION and SPECULATION. These modifications are 


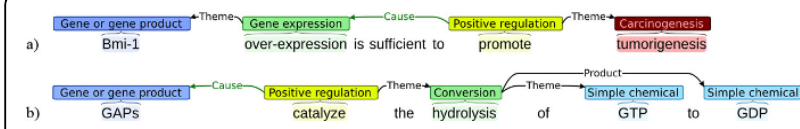

Figure 3 Illustration of event annotations. a) Cancer Genetics task b) Pathway Curation task.

simple binary flags that apply to an event, marking it as being explicitly denied (NEGATION, e.g. "p53 is not affected") or expressed in a speculative context (SPECULATION, e.g. "p53 may be affected"). Event modifications are not associated with a text "trigger", unlike in some related task settings [32,33]. The representation, annotation scope, and semantics of these modifications are defined as in the BioNLP ST'09, similarly to many other subsequent tasks. Event modifications are one of the extraction targets in the two tasks, and systems aiming to address all aspects of the tasks should identify also event NEGATION and SPECULATION.

\section{Data format}

The information introduced above is represented in the tasks using the simple data format first introduced for the BioNLP ST'09. This is a standoff format where the annotated texts are stored separately from their annotations, with annotations referencing relevant spans of text via character offsets. The annotations are further split so that the physical entity annotations that are provided to the task participants also for the test data are stored in one file, and the annotations corresponding to extraction targets in another. Each document thus involves three files: the text, the given annotations, and the extraction targets. The annotations are stored in a line-oriented format where each line contains a single annotation, consisting of a unique identifier, the annotation type, and fields specific to the annotation primitive, such as the trigger and event arguments for event annotations, or the (start, end) character offsets and marked text for physical entity annotations and event triggers. Figure 4 shows a simple example illustrating the data format. For a detailed specification, we refer to the documentation on the shared task homepage [34].

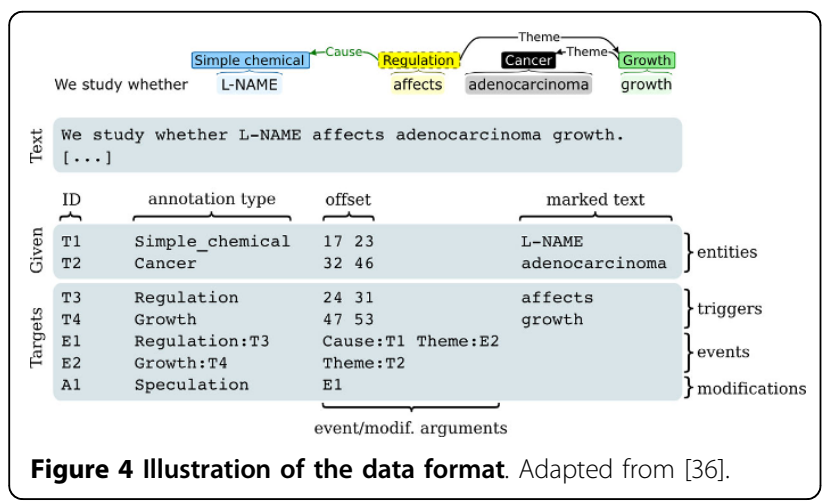

\section{Cancer genetics task setting}

Table 2 summarizes the entity types and reference resources applied in the CG task annotation. Of the molecular level entity types, the most prominent are SIMPLE CHEMICAL, used to mark mentions of the names of non-repetitive chemical entities, and GENE OR GENE PRODUCT, used for the names of genes, proteins, RNA, and their families. These two entity types are closely similar in definition to the CHEMICAL and PROTEIN types included in previous ST tasks [35,36]. However, the CG molecular types are more fine-grained than the corresponding types in these previous tasks, and the task applies detailed types such as PROTEIN DOMAIN OR REGION for cases where many previous tasks used the generic type ENTITY [28].

Anatomy-level entities, a notable novel aspect of the CG task, are subdivided primarily by granularity, and their type labels and definitions mainly follow the Common Anatomy Reference Ontology (CARO) [37], which is a small species-independent ontology based on the Foundational Model of Anatomy (FMA) [38]. For anatomical entities, not only names but also nominal and

Table 2. Cancer Genetics task entity types, reference resources, and definitions

\begin{tabular}{|c|c|c|}
\hline Type & Reference & Ontology ID \\
\hline ORGANISM & $\begin{array}{l}\text { NCBI } \\
\text { taxonomy }\end{array}$ & CARO:0000012 \\
\hline \multicolumn{3}{|l|}{ Anatomical entity } \\
\hline ORGANISM SUBDIVISION & $\begin{array}{l}\text { Species- } \\
\text { specific }\end{array}$ & CARO:0000032 \\
\hline ANATOMICAL SYSTEM & anatomy & CARO:0000011 \\
\hline ORGAN & $\begin{array}{l}\text { resources } \\
\text { (e.g. }\end{array}$ & CARO:0000024 \\
\hline MULTI-TISSUE STRUCTURE & $\begin{array}{l}\text { FMA), } \\
\text { derived }\end{array}$ & CARO:0000055 \\
\hline TISSUE & $\begin{array}{l}\text { resources } \\
\text { (e.g. }\end{array}$ & CARO:0000043 \\
\hline $\begin{array}{l}\text { DEVELOPING ANATOMICAL } \\
\text { STRUCTURE }\end{array}$ & UBERON) & UBERON:0005423 \\
\hline CELL & $\mathrm{CL}$ & CARO:0000013 \\
\hline CELLULAR COMPONENT & GO-CC & GO:0005575 \\
\hline ORGANISM SUBSTANCE & FMA etc. & CARO:0000004 \\
\hline $\begin{array}{l}\text { IMMATERIAL ANATOMICAL } \\
\text { ENTITY }\end{array}$ & FMA etc. & CARO:0000007 \\
\hline PATHOLOGICAL FORMATION & - & - \\
\hline CANCER & - & - \\
\hline \multicolumn{3}{|l|}{ Molecular entity } \\
\hline GENE OR GENE PRODUCT & $\begin{array}{l}\text { gene, } \\
\text { protein, }\end{array}$ & SBO:0000246 \\
\hline PROTEIN DOMAIN OR REGION & and related & SBO:0000493 \\
\hline DNA DOMAIN OR REGION & entity DBs & SBO:0000493 \\
\hline SIMPLE CHEMICAL & ChEBI & SBO:0000247 \\
\hline AMINO ACID & ChEBI & CHEBI:33709 \\
\hline
\end{tabular}

The indentation of the types corresponds to is- $a$ relations. The labels in italics are not annotated types but groupings defined only for organization. 
adjectival references (e.g. "mitochondrial") are annotated. We refer to Ohta et al. [39] for more detailed discussion of the anatomical entity type definitions. Finally, the names of organisms and nominal mentions of comparable specificity (e.g. "patients") are annotated as ORGANISM, as in a number of previous tasks.

To assure consistency, resources identifying entities of each relevant type were used for reference during the entity annotation. Mentions of biomolecules are annotated with reference to databases of genes, proteins, their families, and related entities such as Entrez Gene [40], UniProt [41], PFam [42], and CATH [43], and simple chemical entity mentions with reference to ChEBI [44]. Anatomical entity mentions are annotated primarily with reference to species-specific anatomy ontologies such as FMA and derived resources such as UBERON [45]. For cells and cellular components, available cross-species resources such as the Cell Ontology (CL) [46] and the Gene Ontology Cellular Component subontology (COCC) are used. ORGANISM annotations are created with reference to the NCBI taxonomy [47].

The CG event types and their arguments are summarized in Table 3. As in most previously introduced BioNLP ST task settings, the CG task bases its event types primarily on the Gene Ontology (GO) [48]. Specifically, the definitions of event types of the molecular, general and regulation categories follow the corresponding GO-based types in the 2011 GENIA (GE) [35] and Epigenetics and Post-translational Modifications (EPI) [36] tasks. By contrast, event types of the anatomical and pathological categories have not been considered in previous ST settings. As for the other categories, these events are defined with $\mathrm{GO}$ as the primary reference resource whenever possible.

However, there are some categories of processes that are critical for comprehensive analysis of the cancer literature but fall outside of the scope of GO. Most importantly, GO systematically excludes from its scope pathological processes such as carcinogenesis. We address this limitation in two ways. First, we reinterpret GO types such as GROWTH to extend their scope so as to include also pathological entities, so that e.g. "cancer development" is annotated with the GROWTH type. Second, for intrinsically pathological processes such as METASTASIS that lack a close physiological analogue, we introduce a small number of representative high-level event types such as Metastasis. Finally, we introduce the single upper-level event type PLANNED PROCESS to mark processes explicitly involving experimenter action [49]. We refer to [50] for further information on the design of the CG task events.

\section{Pathway curation task setting}

Four entity types are defined in the PC task: the molecular level entity categories SIMPLE CHEMICAL, GENE
OR GENE PRODUCT, and COMPLEX, and the CELLULAR COMPONENT type (Table 4). Of these four, all but COMPLEX are defined also in the CG task (see previous section), and the two tasks share the definitions of these types. The remaining type, COMPLEX, is used to mark the names of molecular entities of non-covalently linked components, which are particularly relevant to the PC task extraction targets.

Table 5 presents the event types annotated in the PC task and their arguments. By contrast to the CG task and the majority of previously introduced BioNLP ST tasks, the PC task applies the Systems Biology Ontology (SBO) as its primary reference ontology for events, only basing a small number of upper-level event types on GO. While the use of SBO for the PC task entity and event definitions assures compatibility with pathway representations on the type level, supporting pathway curation tasks using event extraction further requires the ability to map event structures to complex pathway reactions (and vice versa).

Popular pathway model standards such as SBML and BioPAX differentiate between three basic categories of reaction participants: reactants, products, and modifiers. The former two correspond roughly to reaction inputs and outputs, while the last category identifies entities that have a regulatory effect, such as inhibiting or catalyzing the reaction. While this categorization of pathway reaction participants has some close analogies to the event structures applied in previous shared tasks, some aspects of the event representation require adjustments to assure a systematic and consistent mapping. In the PC task, we interpret event role types as follows: first, Theme is used to annotate participants corresponding to reactants, and Cause is applied together with regulation event types to capture modifiers and their effects (inhibition, catalysis, etc.). Finally, we introduce the new role Product specifically for the purpose of representing the product role in pathway models.

The use of SBO types and event roles defined with respect to the participant categories in pathways makes events and pathway reactions fully isomorphic in theory. However, natural language text does not necessarily explicitly state all participants, so in practice event representations that adhere to the principle that all annotations are bound to specific expressions in text may not always map to complete reaction representations. For example, in a pathway model, a phosphorylation reaction that takes $p 38 \gamma$ as a reactant would have phospho-p38 $\gamma$ as a product. However, as the combination of reactant, reaction type and product is redundant, authors rarely express all three, instead using more concise forms such as " $p 38 \gamma$ is phosphorylated". Figure 5 illustrates the mapping and the difference between idealized, fully explicit statements and less complete forms that frequently 
Table 3. Cancer Genetics task event types and their arguments

\begin{tabular}{|c|c|c|}
\hline Type & Core arguments & Additional arguments \\
\hline \multicolumn{3}{|l|}{ Anatomical } \\
\hline DEVELOPMENT & Theme (Anatomy) & \\
\hline BLOOD VESSEL DEVELOPMENT & Theme?(Anatomy) & AtLoc? \\
\hline GROWTH & Theme (Anatomy) & \\
\hline DEATH & Theme (Anatomy) & \\
\hline CELL DEATH & Theme?(Cell) & \\
\hline BREAKDOWN & Theme (Anatomy) & \\
\hline CELL PROLIFERATION & Theme (Cell) & \\
\hline CELL DIVISION & Theme (Cell) & \\
\hline CELL DIFFERENTIATION & Theme (Cell) & AtLoc? \\
\hline REMODELING & Theme (Tissue) & \\
\hline REPRODUCTION & Theme (Organism) & \\
\hline \multicolumn{3}{|l|}{ Pathological } \\
\hline MUTATION & Theme (GGP) & AtLoc?, Site? \\
\hline CARCINOGENESIS & Theme?(Anatomy) & Atloc? \\
\hline CELL TRANSFORMATION & Theme (Cell) & AtLoc? \\
\hline METASTASIS & Theme?(Anatomy) & TOLOC \\
\hline INFECTION & Theme?(Anatomy), Participant?(Organism) & \\
\hline \multicolumn{3}{|l|}{ Molecular } \\
\hline METABOLISM & Theme (Molecule) & \\
\hline SYNTHESIS & Theme (Simple chemical) & \\
\hline CATABOLISM & Theme (Molecule) & \\
\hline AMINO ACID CATABOLISM & Theme?(Molecule) & \\
\hline GLYCOLYSIS & Theme?(Molecule) & \\
\hline GENE EXPRESSION & Theme+(GGP) & \\
\hline TRANSCRIPTION & Theme (GGP) & \\
\hline TRANSLATION & Theme (GGP) & \\
\hline PROTEIN PROCESSING & Theme (GGP) & \\
\hline PHOSPHORYLATION & Theme (Molecule) & Site? \\
\hline \multicolumn{3}{|c|}{ (other chemical modifications defined similarly to PHOSPHORYLATION) } \\
\hline PATHWAY & Participant (Molecule) & \\
\hline \multicolumn{3}{|l|}{ General } \\
\hline BINDING & Theme+(Molecule) & Site? \\
\hline DISSOCIATION & Theme (Molecule) & Site? \\
\hline LOCALIZATION & Theme+(Molecule) & At/From/ToLoc? \\
\hline Regulation & Theme (Any), Cause?(Any) & \\
\hline POSITIVE REGULATION & Theme (Any), Cause?(Any) & \\
\hline NEGATIVE REGULATION & Theme (Any), Cause?(Any) & \\
\hline PLANNED PROCESS & Theme*(Any), Instrument*(Entity) & \\
\hline
\end{tabular}

The indentation corresponds to ontological structure (is-a/part-of relations). The suffixes ? ${ }^{*}$, , and + denote zero or one, zero or more, and one or more arguments of the shown type (respectively). GGP stands for Gene or gene product. For brevity, additional argument types are not shown in table: the AtLoc, FromLoc and ToLoc arguments take an anatomical entity type, and Site arguments take a Protein domain or region or DNA domain or region entity type.

appear in actual publications. We refer to [24] for further discussion of the relation between the PC task entity and event types, the event representation, and representations applied in pathway models.

\section{Corpora}

Both the Cancer Genetics and Pathway Curation tasks use corpus resources based in part on relevant
Table 4. Pathway Curation task entity types, reference resources, and definitions

\begin{tabular}{lll}
\hline Entity type & Reference & Ontology ID \\
\hline SIMPLE CHEMICAL & ChEBI & SBO:0000247 \\
GENE OR GENE PRODUCT & gene/protein DBs & SBO:0000246 \\
COMPLEX & complex DBs & SBO:0000253 \\
CELLULAR COMPONENT & GO-CC & SBO:0000290 \\
\hline
\end{tabular}


Table 5. Pathway Curation task event types and arguments

\begin{tabular}{|c|c|c|c|}
\hline Event type & Core arguments & Additional arguments & Ontology ID \\
\hline CONVERSION & Theme(Molecule), Product(Molecule) & & SBO:0000182 \\
\hline PHOSPHORYLATION & Theme(Molecule), Cause(Molecule) & Site(Simple chemical) & SBO:0000216 \\
\hline DEPHOSPHORYLATION & Theme(Molecule), Cause(Molecule) & Site(Simple chemical) & SBO:0000330 \\
\hline \multicolumn{4}{|c|}{ (Other modifications, such as ACETYLATION, defined similarly.) } \\
\hline LOCALIZATION & Theme(Molecule) & At/From/ToLoc(Cell. comp.) & GO:0051179 \\
\hline TRANSPORT & Theme(Molecule) & From/ToLoc(Cell. comp.) & SBO:0000185 \\
\hline GENE EXPRESSION & Theme(Gene or gene product) & & GO:0010467 \\
\hline TRANSCRIPTION & Theme(Gene or gene product) & & SBO:0000183 \\
\hline TRANSLATION & Theme(Gene or gene product) & & SBO:0000184 \\
\hline DEGRADATION & Theme(Molecule) & & SBO:0000179 \\
\hline BINDING & Theme(Molecule), Product(Complex) & & SBO:0000177 \\
\hline DISSOCIATION & Theme(Complex), Product(Molecule) & & SBO:0000180 \\
\hline REGULATION & Theme(Any), Cause(Any) & & GO:0065007 \\
\hline \multirow[t]{2}{*}{ POSITIVE REGULATION } & Theme(Any), Cause(Any) & & GO:0048518, \\
\hline & & & GO:0044093 \\
\hline ACTIVATION & Theme(Molecule), Cause(Any) & & SBO:0000412 \\
\hline \multirow[t]{2}{*}{ NEGATIVE REGULATION } & Theme(Any), Cause(Any) & & GO:0048519, \\
\hline & & & GO:0044092 \\
\hline INACTIVATION & Theme(Molecule), Cause(Any) & & SBO:0000412 \\
\hline PATHWAY & Participant(Molecule) & & SBO:0000375 \\
\hline
\end{tabular}

"Molecule" represents any of Simple chemical, Gene or gene product, or Complex. "Any" refers to an annotation of any type. The indentation of the types corresponds to ontological relations (is- $a$ and part-of) between the event types

previously released corpora. Nevertheless, most of the annotations in the shared task versions of the training, development and test corpora of both tasks were newly introduced specifically for these tasks. The following sections present these resources and their preparation.

\section{Annotation process}

The annotation of the CG and PC task corpora followed the same overall process: document selection, automatic pre-annotation of entity mentions, manual finalization of entity annotations, and manual event annotation.

While some of the aspects of the entity annotation are novel, many of the annotated entity types are in scope of established domain tools and resources. To reduce the overall annotation effort, we thus created preliminary annotation using a selection of automatic named entity and entity mention taggers. For SIMPLE CHEMICAL

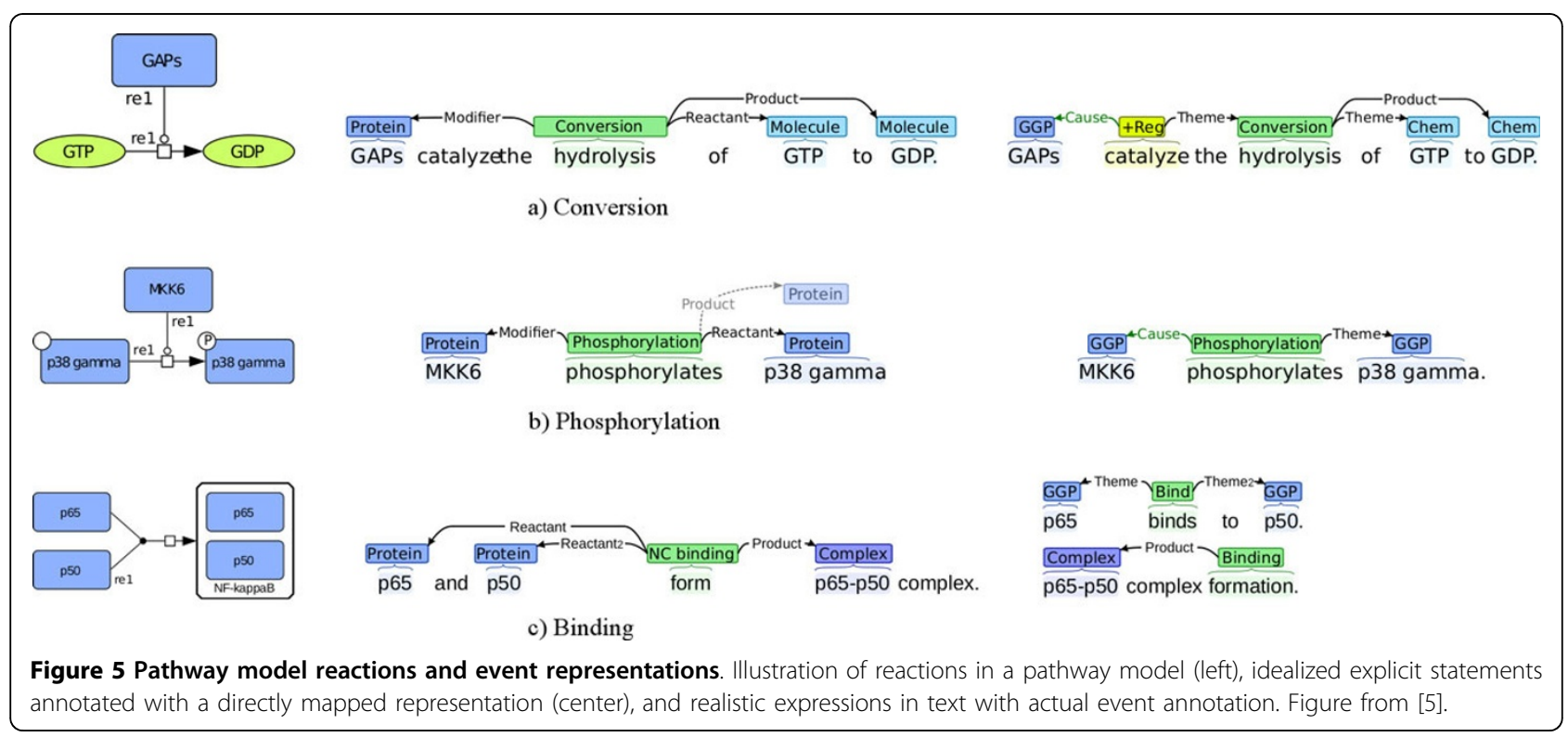


tagging, we used the OSCAR4 system, which was trained on the chemical entity mention recognition corpus of Corbett and Copestake [51]. For GENE OR GENE PRODUCT mention detection, we used BANNER [52] for the CG task and NERsuite [53] for the PC task. Both of these systems were trained on the Gene Mention task corpus introduced in the BioCreative 2 evaluation [54]. NERsuite was also applied for anatomical entity mention detection (CULLULAR COMPONENT only for the PC task). For these tagging tasks, the general machine learningbased system was trained on the Anatomical Entity Mention (AnEM) corpus [39] following the approach presented by Pyysalo and Ananiadou [55]. As no broad-coverage corpus annotated specifically for mentions of macromolecular complexes was available, we applied heuristics based on the GENE OR GENE PRODUCT annotation and dictionarybased tagging to create the initial annotations for the PC task COMPLEX type. Finally, LINNAEUS [56] was applied for the CG task ORGANISM mentions. The overall processing used the pipeline first introduced for similar analysis for the BioNLP ST'11 [57]. These tools were additionally integrated into the Argo workflow system [58] to support the PC task curation process. Following initial automatic entity mention annotation, we performed manual revision of the outputs to correct tagger errors prior to advancing to the event annotation stage.

We acknowledge that automatic annotation is not only far from perfect, but also carries a risk of introducing systematic errors, some of which may persist through subsequent manual revision. As entity annotations were not a target of extraction in either of the tasks, the possibility of some remaining bias from such errors was considered acceptable. By contrast, we wished to assure that the quality of the event annotations was as high as possible and to avoid any possibility of introducing systematic errors that might call into question whether the evaluation provides a fair representation of the comparative performance of different extraction approaches. For this reason, the event annotation of both tasks was created manually from scratch, forgoing any initial automatic annotation.

All manual annotation, including the revision of the initial automatic entity mention annotations as well as the primary event annotation, was performed using the open source BRAT annotation tool [59].

The task-specific annotation process details are presented below.

\section{Cancer genetics corpus annotation}

The CG corpus consists of 600 PubMed abstracts selected on the basis of their relevance to established hallmarks of cancer, such as the evasion of apoptosis, tissue invasion, and sustained angiogenesis [6]. Of these 600 documents, 250 are part of the previously released multi-level event extraction (MLEE) corpus [50], which concentrates specifically on the angiogenesis subdomain. The other 350 documents were selected by querying PubMed for MeSH terms that relate to other hallmarks of cancer, such as apoptosis and metastasis (Table 6). After identifying queries providing sufficient specificity, we selected a random sample of the resulting documents and performed a round of manual filtering to assure that each abstract is relevant to the selected characteristic of cancer and its molecular basis.

The CG corpus was annotated by Tomoko Ohta, a PhD biologist with a cancer molecular biology background and extensive experience in event annotation. The creation of the anatomical entity annotation is described in [55], and the event annotation extended the guidelines and manual annotation process introduced in [50].

After the completion of the initial manual annotation, we performed some iterations of automatically supported revisions to further improve annotation quality and consistency. For example, we used the consistency check options of the search.py script included with BRAT to find text strings that were annotated as entity mentions in some cases but not in others and annotations that shared the same surface form but were assigned different semantic types. Automatically detected potential inconsistencies were subjected to further manual examination and correction when necessary. As only a single annotator was trained to perform the CG corpus annotation, we did not directly measure inter-annotator agreement during the annotation of this corpus. However, our previous IAA evaluation using the same annotation protocol [50] indicated that following an initial training round, the anatomical entity annotation could be replicated by a second annotator with $94.1 \%$ accuracy and the event annotation with $72.5 \%$ F-score using the primary task evaluation criteria.

\section{Pathway curation corpus annotation}

As for the CG task, the texts of the PC task corpus are also drawn from PubMed abstracts. For the CG corpus, we aimed to assure that each included abstract provides evidence for the annotation of some reaction found in at least one selected pathway model (Table 7). To select such documents, we applied two complementary strategies, one predicated on the presence of explicit references to evidence documents in the pathway annotations, and the other using a system developed specifically to support the creation of such annotations.

For the first approach, we selected the BioModels pathways that had the most manually annotated references identifying a document in PubMed. We then retrieved the abstracts of a random sample of these documents and filtered them manually to assure their relevance to the task. The second strategy was required as the majority of pathway models do not include any references to evidence documents. To select documents providing evidence for 
Table 6. Queries for Cancer Genetics task document selection

\begin{tabular}{lll}
\hline Domain & Documents & Query terms \\
\hline Carcinogenesis & 150 & cell transformation, neoplastic AND (proteins OR genes) \\
Metastasis & 100 & neoplasm metastasis AND (proteins OR genes) \\
Apoptosis & 50 & apoptosis AND (proteins OR genes) \\
Glucose metabolism & 50 & (glucose/metabolism OR glycolysis) AND neoplasms \\
\hline
\end{tabular}

Only matches against MeSH terms were queried for, excluding cases where the query terms appeared only in text (e.g. "neoplasms"[MeSH Terms]).

reactions in other pathways, we entered selected PANTHER DB models into PathText [60], a system for identifying documents relevant to specific reactions. We then selected random reactions from these models and manually filtered the top results retrieved by PathText for relevance. This approach is described in detail by Miwa et al. [61].

The PC task annotation effort was carried out in collaboration between the UK National Centre for Text Mining $(\mathrm{NaCTeM})$ and the Korea Institute of Science and Technology Information (KISTI). The entity annotation followed the automatically supported approach described above, and event annotation was performed fully manually by three biologists. The effort was organized by a coordinator with experience from several event annotation projects, but the individual annotators had no previous experience with the annotation of natural language texts. After a brief introduction to event annotation and training with the annotation tool and guidelines, the primary annotation was performed otherwise independently, but with a random $20 \%$ of documents provided to all three annotators to evaluate consistency, identify points of disagreement, and

Table 7. Pathway models used to select documents for the Pathway Curation task

\begin{tabular}{llll}
\hline Pathway & Repository & ID & Publication \\
\hline mTOR & BioModels & MODEL1012220002 & {$[88]$} \\
mTORC1 upstream & BioModels & MODEL1012220003 & {$[88]$} \\
regulators & & & \\
TLR & BioModels & MODEL2463683119 & {$[89]$} \\
Yeast Cell Cycle & BioModels & MODEL1011020000 & {$[90]$} \\
Rb & BioModels & MODEL4132046015 & {$[91]$} \\
EGFR & BioModels & MODEL2463576061 & {$[92]$} \\
Human Metabolic & BioModels & MODEL6399676120 & {$[93]$} \\
Network & & & \\
NF-kappaB pathway & - & - & {$[23]$} \\
p38 MAPK & PANTHER & P05918 & - \\
& DB & & \\
p53 & PANTHER & P00059 & - \\
& DB & & - \\
p53 feedback loop & PANTHER & P04392 & - \\
pathway & DB & & - \\
Wnt signaling pathway & PANTHER & P00057 & \\
& DB & & \\
\hline
\end{tabular}

measure inter-annotator agreement. Following the completion of the primary manual annotation, consistency checking using automatic methods to detect potentially inconsistent annotations was performed similarly as in the CG task data preparation (described above).

An evaluation of the redundantly annotated documents after consistency checking indicated an inter-annotator agreement of $61.0 \% \mathrm{~F}$-score for the event annotation using the primary task evaluation criteria. This level of agreement is somewhat low given the comparative simplicity of the task annotation targets. This can likely be attributed in substantial part to the facts that the annotators were working in a different country from the annotation coordinators, communicating primarily over email, and that only a relatively short period of annotator training could be included due to the overall project schedule. This serves to emphasize that while it is necessary to involve domain experts in annotation efforts targeting specialized texts, such expertise must be accompanied by thorough annotator training that provides sufficient experience on the use of the annotation formalism and tools as well as detailed understanding of the annotation guidelines. Finally, the redundantly annotated documents were assessed by the annotation coordinator to select the best of each set of annotations for the final corpus. This selection implies that the measured inter-annotator agreement of $61 \% \mathrm{~F}$-score is a lower bound on the quality of the released PC task event annotation.

\section{Corpus statistics}

Both the CG and PC task corpora were divided into training, development and test sets representing approximately $50 \%, 17 \%$, and $33 \%$ of the documents, respectively. Table 8 summarizes the CG corpus statistics, and Table 9 gives the statistics of the PC corpus. We note that the CG corpus is the largest of the BioNLP ST 2013 corpora by most of these measures, including in particular the number of annotated events. While the PC task corpus is somewhat smaller, it nevertheless has more annotated events than all corpora in previous BioNLP ST tasks with the exception of the original GE task. We thus expect that the availability of sufficient numbers of training examples should not be a limiting factor for the performance of systems participating in either task compared to previous BioNLP ST tasks. 
Table 8. Cancer Genetics task corpus statistics

\begin{tabular}{lllll}
\hline Item & Train & Devel & Test & Total \\
\hline Documents & 300 & 100 & 200 & 600 \\
Words & 66082 & 21732 & 42064 & 129878 \\
Entities & 11034 & 3665 & 6984 & 21683 \\
Relations & 466 & 176 & 275 & 917 \\
Events & 8803 & 2915 & 5530 & 17248 \\
Modifications & 670 & 214 & 442 & 1326 \\
\hline
\end{tabular}

\section{Evaluation}

The evaluation of both the CG and PC tasks is instancebased and uses the standard precision, recall and F-score metrics. The primary criteria for determining if an event (or event modification) predicted by a participating system matches a gold standard event (modification) follow those first introduced in the initial BioNLP ST. In brief, these criteria require that a predicted and gold event are identical with two possible exceptions: the boundaries of predicted events may differ from those of the corresponding gold event by up to one word on each side, and the events that are referred to as arguments of the predicted and gold events under consideration may differ in their non-core arguments (see Tables 3 and 5). We refer to Kim et al. [28] for a detailed definition of these criteria. The statistical significance of the performance differences between each pair of participating systems was assessed using the approximate randomization method $[62,63]$ using 9,999 repetitions.

We consider here also two variants of the primary evaluation setting: evaluation on core extraction targets only, and evaluation using an additional relaxation of the matching criteria termed single partial penalty. For the core task evaluation, we removed from both the gold annotation and the predictions of each participant all but the core event arguments (Tables 3 and 5) as well as event modifications, and then eliminated duplicate events arising from this simplification. This evaluation setting corresponds to the GE task subtask 1 [35] and the core evaluation settings of the EPI and Infectious Diseases (ID) tasks [36], focusing evaluation on a reduced set of targets defining only the minimum required to characterize an event. The single partial penalty criterion, first proposed in [64], aims to address the following issue in the

Table 9. Pathway Curation task corpus statistics

\begin{tabular}{lllll}
\hline Item & Train & Devel & Test & Total \\
\hline Documents & 260 & 90 & 175 & 525 \\
Words & 53811 & 18579 & 35966 & 108356 \\
Entities & 7855 & 2734 & 5312 & 15901 \\
Relations & 455 & 128 & 330 & 913 \\
Events & 5992 & 2129 & 4004 & 12125 \\
Modifications & 317 & 80 & 174 & 571 \\
\hline
\end{tabular}

primary evaluation criteria: events predicted by a system that otherwise match a gold standard event but either lack some of the arguments of that event or have some extra arguments are penalized twice: the predicted event counts as a false positive, and the gold standard event it largely corresponds to counts as a false negative. With the single partial penalty criterion, when a prediction and a gold standard event match partially in this way, either the prediction is counted as a false positive (extra arguments) or the gold standard event as a false negative (missing arguments), but not both. Finally, we consider also the combination of the core evaluation and single partial penalty variants.

\section{Results and discussion \\ Participation}

Final results for the two tasks were submitted by six teams, with all six submitting to the CG task and two submitting also to the PC task. Table 10 presents a summary of the teams, their members, and the ranks of their systems at the tasks they participated in. These teams involved members from six academic groups as well as one company, representing a broad variety of backgrounds, including linguists, bioinformaticians, and computer scientists among others.

Table 11 summarizes the key properties of the systems applied to the two tasks. The participants approached the tasks using a broad variety of different architectures, including one-best machine learning pipelines (TEES-2.1 and $\mathrm{NaCTeM}$ ), a joint subgraph matching-based approach (NCBI), a rule-based method (RelAgent), and two parsing-based approaches (UET-NII and ISI). Machine learning-based processing stages are dominated by the application of support vector machines, a popular learning method also in previous shared tasks.

A range of different methods was applied also in support of the individual processing stages. The methods used for basic word-level processing are generally comparatively simple, domain-independent approaches, with only the NCBI system using a lemmatization method developed specifically for domain texts (BioLemmatizer) [65]. By contrast, we note that all six systems perform syntactic analysis using primarily advanced, domain-specific parsing methods. These choices may be motivated in part by the observation that the application of parsers has been a very common feature of high-performing systems in previous BioNLP ST events. However, there is substantial variety in the specific choice of parser. The TEES-2.1 and NCBI systems use the McClosky-Charniak-Johnson constituency parser $[66,67]$ and the Stanford Dependency conversion [68], which was the most common choice for syntactic analysis in BioNLP ST'11. The systems of NaCTeM and UET-NII use the probabilistic HPSG parser Enju [69], with $\mathrm{NaCTeM}$ additionally using the GDep shift-reduce 
Table 10. Participating teams, ranks and references to system descriptions

\begin{tabular}{|c|c|c|c|c|}
\hline Team & Institution & Tasks (rank) & Members & Ref \\
\hline TEES-2.1 & University of Turku & $C G(1), P C(2)$ & $1 \mathrm{Bl}$ & {$[94,95]$} \\
\hline $\mathrm{NaCTeM}$ & National Centre for Text Mining & $P C(1), C G(2)$ & $1 \mathrm{NLP}$ & {$[96,97]$} \\
\hline $\mathrm{NCBI}$ & National Center for Biotechnology Information & CG(3) & $3 \mathrm{Bl}$ & {$[98,99]$} \\
\hline RelAgent & RelAgent Private Ltd. & $C G(4)$ & $1 \mathrm{LI}, 1 \mathrm{CS}$ & {$[71]$} \\
\hline UET-NII & University of Engineering and Technology, Vietnam and National Institute of Informatics, Japan & $C G(5)$ & $6 \mathrm{CS}$ & [100] \\
\hline$|S|$ & Indian Statistical Institute & $C G(6)$ & $2 \mathrm{ML}, 2 \mathrm{NLP}$ & - \\
\hline
\end{tabular}

Abbreviations: BI: Bioinformatician, CS: Computer Scientist, LI: Linguist, ML: Machine Learning researcher, NLP: Natural Language Processing researcher.

dependency parser [70]. Finally, the Stanford CoreNLP tools are used by the ISI system, and a custom parser is applied by RelAgent [71].

Although event modifications were included as an extraction target in both tasks, three of the six systems involve no event modification component at all. Of the three that do, two apply a machine learning approach and one a rule-based approach. The choice of the three participants to exclude event modification from their methods may reflect at least in part the rarity of modifications compared to event annotations (see Tables 8 and 9) and the known difficulty of this subtask.

As in previous instantiations of the BioNLP ST, the 2013 shared task was run as an open challenge, where participants were encouraged to use any additional resources to augment the training data provided by the task organizers. Of such external resources, perhaps the most obviously applicable is the set of corpora introduced in previous shared tasks: as discussed in the introduction, these tasks have involved many of the same extraction targets, in particular basic biomolecular events. Three teams made use of one or more previously introduced BioNLP ST corpora. The TEES-2.1 and NCBI teams both used the GE corpus [35] and NCBI used also the EPI task corpus [36]. The $\mathrm{NaCTeM}$ system did not use any external corpora for the CG task, but for the PC task the system was applied with a stacked model [72] with predictions also from models trained on the BioNLP ST'11 GE, EPI and ID tasks [36] as well as from four event corpora not included in a shared task [24,73-75]. Three teams also applied lexical resources based on event corpora (Table 11).

We note that of the eight submissions received from the six participating groups, five represent applications or extensions of previously proposed approaches: the University of Turku and the National Centre for Text Mining submitted to both tasks using their established, state-of-the-art event extraction systems, the Turku Event Extraction System [76,77] (TEES) and EventMine $[78,79](\mathrm{NaCTeM})$, and the National Center for Biotechnology Information participated in the CG task using an extension of their previously proposed subgraph matching approach $[80,81]$. By contrast, the CG task submissions from the RelAgent, UET-NII and ISI teams used approaches that were now evaluated in a BioNLP ST event for the first time.

For more information on the participating systems, we refer to the descriptions of these systems published by the groups who created them, summarized in Table 10.

\section{Cancer genetics primary evaluation results}

The Cancer Genetics task primary evaluation results are presented in summary in Table 12. Statistical significance testing indicated that all pairwise differences between systems are significant $(p<0.05)$ excepting for that between the NCBI and RelAgent systems.

The TEES-2.1 system achieved the best performance at the task, averaging an F-score of 55\%. A version of the same system ranked first also in the original 2009 BioNLP

Table 11. Summary of system architectures

\begin{tabular}{|c|c|c|c|c|c|c|c|c|}
\hline \multirow[b]{2}{*}{ Team } & \multicolumn{2}{|c|}{ NLP methods } & \multicolumn{4}{|c|}{ Events } & \multicolumn{2}{|c|}{ Resources } \\
\hline & Lexical & Syntactic & Trigger & Arg & Group & Modif. & Corpora & Other \\
\hline TEES-2.1 & Porter & $\mathrm{MCCCJ}+\mathrm{SD}$ & SVM & SVM & SVM & SVM & GE & hedge words \\
\hline $\mathrm{NaCTeM}$ & Snowball & Enju, GDep & SVM & SVM & SVM & SVM & (see text) & triggers \\
\hline $\mathrm{NCBI}$ & MedPost, BLem & $\mathrm{MCCCJ}+\mathrm{SD}$ & \multicolumn{3}{|c|}{ Joint, subgraph matching } & - & GE, EPI & - \\
\hline RelAgent & Brill & fnTBL, custom & rules & rules & rules & rules & - & - \\
\hline UET-NII & Porter & Enju & SVM & MaxEnt & Earley & - & - & triggers \\
\hline$|S|$ & CoreNLP & CoreNLP & NERsuite & \multicolumn{2}{|c|}{ Joint, MaltParser } & - & - & - \\
\hline
\end{tabular}

Abbreviations: Trigger: event trigger detection, Arg: trigger-argument relation detection, Group: argument grouping into event structures, Modif.: event modification prediction, CoreNLP: Stanford CoreNLP, Porter: Porter stemmer, BLem: BioLemmatizer, Snowball: Snowball stemmer, McCCJ: McClosky-CharniakJohnson parser, Charniak: Charniak parser, SD: Stanford Dependency conversion, SVM: Support Vector Machines, MaxEnt: Maximum Entropy Modeling. 
Table 12. Cancer Genetics task primary evaluation result summary

\begin{tabular}{llll}
\hline Team & recall & prec. & F-score \\
\hline TEES-2.1 & 48.76 & $\mathbf{6 4 . 1 7}$ & $\mathbf{5 5 . 4 1}$ \\
NaCTeM & $\mathbf{4 8 . 8 3}$ & 55.82 & 52.09 \\
NCBI & 38.28 & 58.84 & 46.38 \\
RelAgent & 41.73 & 49.58 & 45.32 \\
UET-NII & 19.66 & 62.73 & 29.94 \\
ISI & 16.44 & 47.83 & 24.47 \\
\hline
\end{tabular}

ST, then achieving an F-score of 52\% [82], as well as in four out of eight tasks in the 2011 shared task, there achieving an F-score of 53\% in the comparable GE task [83]. The NaCTeM group ranked second at the CG task, applying the EventMine system that has also been evaluated on numerous previous shared task corpora. The performance it achieves here, an F-score of 52\%, is likewise at a broadly similar level to its performance at previously introduced tasks [84]. Also the third-ranking group, NCBI, extends on a system previously applied at the BioNLP ST'11, here performing notably better in relative terms at an F-score of $46 \%$ than the F-score of $41 \%$ achieved in the 2011 GE task [80].

Of the three newly introduced systems, the rule-based system of RelAgent achieves the best performance, averaging an F-score of $46 \%$ and thus performing within 10 percentage points of the top-ranking system. This shows that the CG task targets are not so complicated as to make the task inaccessible to rule-based approaches and that newly introduced systems can achieve a level of performance that is broadly competitive with the established state-of-the-art systems. The parsing-based systems achieve less competitive results, with F-scores of 30\% for UET-NII and $24 \%$ for ISI. The results for both systems are primarily limited by low levels of recall, suggesting that better F-scores could perhaps be achieved by trading off some of the comparatively high precision.

We thus observe that the top three positions are taken by previously established systems - perhaps not a surprising finding - and, more importantly, that each of these systems achieves a level of performance at the CG task that is broadly comparable or better than the performance of the same system at previous related and narrower tasks such as GE. This is highly encouraging as it indicates that the state-of-the-art systems generalize to meet all of the novel challenges of the CG task, including the substantially increased number of entity and event types, the cancer domain, as well as the inclusion of higher levels of biological organization.

Table 13 gives the primary results separately for each event type. These results show that the newly introduced anatomical and pathological event categories are not particularly challenging for the event extraction methods; indeed, the best results for anatomical category events are better than the best results for molecular events (F-score of 77\% versus 73\%). The best results for pathological processes are only slightly lower at an F-score of $68 \%$. By contrast to the broad new categories of events, the specific newly introduced event type Planned process PLANNED PROCESS proved challenging to extract (best result was an F-score of 41\%), perhaps in part due to the fact that it frequently involves multiple arguments.

The previously established event categories general and regulation remain as challenging here as in previous related challenges, likely reflecting at least partly the known challenges in the extraction of events with multiple arguments (e.g. BINDING) and those that recursively include other events (regulation types). Results are comparatively low also for the modification types, with a best F-score of $40 \%$ for NEGATION and 30\% for SPECULATION. The extraction of these types involve challenges similar to those for regulation events in that their correct extraction requires also the correct extraction of the events that they apply to [28].

\section{Pathway curation primary evaluation results}

The overall results of the PC task are given in Table 14. The $\mathrm{NaCTeM}$ team achieves the better performance on the task, an F-score of 53\%, using the EventMine system. The TEES-2.1 system comes in a close second at an F-score of $51 \%$. Although these F-scores do not differ much, the two systems arrive at these results with quite different predictions: while the $\mathrm{NaCTeM}$ system exhibits balanced precision and recall, the performance of TEES2.1 is noticeably skewed in favor of higher precision and lower recall.

Table 15 shows the results of the PC task separated by event type. The two systems show very similar patterns of performance in terms of F-scores, again with the $\mathrm{NaCTeM}$ approach reaching its results with reasonably balanced precision and recall results for most types and TEES-2.1 frequently showing a clear preference for precision over recall. The performance for different event categories shows mainly similar patterns as in previous molecular-level event extraction tasks: simple modification events such as PHOSPHORYLATION that frequently take only a single Theme argument are comparatively easy extraction targets (a better result of an F-score of $66 \%$ ), while structures that require the correct extraction of events that they apply to are challenging (40\% F-score for regulation and $29 \%$ for modifications). Interestingly, the general category of events, and in particular the previously often particularly demanding BINDING type show comparatively high results here, perhaps reflecting some special characteristics of the domain.

It is interesting that the ranks of the two systems are inverted here compared to the CG task, where the 
Table 13. Cancer Genetics task primary evaluation F-scores by event type

\begin{tabular}{|c|c|c|c|c|c|c|}
\hline Event & TEES-2.1 & $\mathrm{NaCTeM}$ & $\mathrm{NCBI}$ & RelAgent & UET-NII & ISI \\
\hline DEVELOPMENT & 71.43 & 64.77 & 67.33 & 66.31 & 61.72 & 53.66 \\
\hline BLOOD VESSEL DEVELOPM & 85.28 & 78.82 & 81.92 & 79.60 & 21.49 & 13.56 \\
\hline GROWTH & 75.97 & 59.85 & 66.67 & 76.92 & 70.87 & 65.52 \\
\hline DEATH & 81.74 & 73.17 & 74.07 & 64.71 & 77.78 & 63.16 \\
\hline CELL DEATH & 73.30 & 75.18 & 78.05 & 66.98 & 25.17 & 7.35 \\
\hline CELL PROLIFERATION & 80.00 & 78.33 & 72.73 & 64.39 & 71.43 & 57.40 \\
\hline CELL DIVISION & 0.00 & 0.00 & 0.00 & 0.00 & 0.00 & 0.00 \\
\hline CELL DIFFERENTIATION & 56.34 & 48.48 & 48.98 & 54.55 & 59.26 & 24.14 \\
\hline REMODELING & 30.00 & 22.22 & 21.05 & 40.00 & 20.00 & 23.53 \\
\hline REPRODUCTION & 100.00 & 100.00 & 100.00 & 100.00 & 100.00 & 100.00 \\
\hline Anatomical total & 77.20 & 71.31 & 73.68 & 70.82 & 50.04 & 38.86 \\
\hline MUTATION & 38.00 & 41.05 & 25.11 & 27.36 & 27.91 & 9.52 \\
\hline CARCINOGENESIS & 77.94 & 72.18 & 67.14 & 64.12 & 35.96 & 24.72 \\
\hline CELL TRANSFORMATION & 81.56 & 82.54 & 71.13 & 67.07 & 57.14 & 32.39 \\
\hline BREAKDOWN & 76.74 & 70.13 & 76.54 & 42.42 & 58.67 & 50.70 \\
\hline METASTASIS & 70.91 & 51.05 & 52.69 & 47.79 & 56.41 & 26.20 \\
\hline INFECTION & 69.57 & 76.92 & 69.23 & 33.33 & 11.76 & 0.00 \\
\hline Pathological total & 67.51 & 59.78 & 54.19 & 48.14 & 46.90 & 25.17 \\
\hline METABOLISM & 83.87 & 70.27 & 74.29 & 80.00 & 68.75 & 71.43 \\
\hline SYNTHESIS & 78.26 & 71.11 & 78.26 & 53.57 & 64.71 & 48.65 \\
\hline CATABOLISM & 63.64 & 36.36 & 38.10 & 23.08 & 20.00 & 36.36 \\
\hline GLYCOLYSIS & 0.00 & 100.00 & 95.45 & 97.78 & 0.00 & 0.00 \\
\hline AMINO ACID CATABOLISM & 0.00 & 66.67 & 66.67 & 66.67 & 0.00 & 0.00 \\
\hline GENE EXPRESSION & 78.21 & 79.96 & 73.69 & 69.45 & 58.01 & 53.28 \\
\hline TRANSCRIPTION & 37.33 & 42.86 & 51.55 & 28.12 & 32.00 & 20.93 \\
\hline TRANSLATION & 40.00 & 22.22 & 0.00 & 0.00 & 0.00 & 0.00 \\
\hline PROTEIN PROCESSING & 100.00 & 100.00 & 100.00 & 0.00 & 100.00 & 100.00 \\
\hline ACETYLATION & 100.00 & 100.00 & 66.67 & 100.00 & 66.67 & 66.67 \\
\hline GLYCOSYLATION & 100.00 & 100.00 & 100.00 & 100.00 & 100.00 & 100.00 \\
\hline PHOSPHORYLATION & 63.33 & 70.37 & 53.12 & 64.15 & 58.33 & 50.00 \\
\hline UBIQUITINATION & 100.00 & 100.00 & 0.00 & 100.00 & 0.00 & 100.00 \\
\hline DEPHOSPHORYLATION & 0.00 & 80.00 & 100.00 & 100.00 & 0.00 & 0.00 \\
\hline DNA METHYLATION & 66.67 & 66.67 & 30.30 & 42.11 & 32.43 & 33.33 \\
\hline DNA DEMETHYLATION & 0.00 & 0.00 & 0.00 & 0.00 & 0.00 & 0.00 \\
\hline PATHWAY & 71.30 & 59.07 & 51.14 & 34.29 & 18.31 & 35.64 \\
\hline Molecular total & 72.60 & 72.77 & 67.33 & 60.72 & 49.35 & 46.70 \\
\hline BINDING & 45.35 & 43.93 & 37.89 & 32.69 & 33.94 & 11.92 \\
\hline DISSOCIATION & 0.00 & 0.00 & 0.00 & 0.00 & 0.00 & 0.00 \\
\hline LOCALIZATION & 54.83 & 57.20 & 47.58 & 45.22 & 44.94 & 35.94 \\
\hline General total & 52.20 & 53.08 & 44.70 & 40.89 & 41.76 & 29.59 \\
\hline REGULATION & 32.66 & 28.73 & 14.19 & 26.48 & 5.51 & 4.57 \\
\hline POSITIVE REGULATION & 45.89 & 44.18 & 34.70 & 38.40 & 13.00 & 12.33 \\
\hline NEGATIVE REGULATION & 47.79 & 43.17 & 33.20 & 40.47 & 10.30 & 12.16 \\
\hline Regulation total & 43.08 & 39.79 & 29.21 & 35.58 & 10.30 & 10.29 \\
\hline PLANNED PROCESS & 39.43 & 40.51 & 34.28 & 28.57 & 22.74 & 21.22 \\
\hline Sub-total & 56.75 & 53.50 & 48.56 & 46.37 & 31.72 & 25.90 \\
\hline NEGATION & 40.00 & 29.55 & 0.00 & 34.64 & 0.00 & 0.00 \\
\hline SPECULATION & 27.14 & 30.35 & 0.00 & 25.90 & 0.00 & 0.00 \\
\hline Modification total & 34.66 & 29.95 & 0.00 & 30.88 & 0.00 & 0.00 \\
\hline Total & 55.41 & 52.09 & 46.38 & 45.32 & 29.94 & 24.47 \\
\hline
\end{tabular}


Table 14. Pathway Curation task primary evaluation result summary

\begin{tabular}{llll}
\hline Team & recall & prec. & F-score \\
\hline $\mathrm{NaCTeM}$ & 52.23 & 53.48 & 52.84 \\
TEES-2.1 & 47.15 & 55.78 & 51.10 \\
\hline
\end{tabular}

TEES-2.1 system achieved 3 percentage points higher performance than EventMine. The only notable difference in the way that the systems were applied in the two tasks is that the EventMine system was trained using a large number of previously introduced resources for the PC but not the CG task [72]. Although the differences in relative performance may be due in part also to other factors, this is a promising indication that the use of previously introduced event resources in training may be beneficial for this extraction task despite its differences from those resources.
As discussed in detail above, both EventMine and TEES are well-established, support vector machinebased pipeline systems that have demonstrated state-ofthe-art performance across many previous event extraction tasks $[78,83]$. Thus, even though the PC task attracted only limited participation at the BioNLP ST'13, the finding that these two highly competitive systems perform in the $51-53 \% \mathrm{~F}$-score range suggests that this is probably a reasonable estimate of the best performance that current event extraction systems can achieve at this task.

It is perhaps somewhat surprising that the best results for this purely molecular-level task are lower than those for the considerably broader CG task despite the same systems achieving the best results in both. However, it is nevertheless a positive finding that this newly introduced task employing a novel reference ontology and aligned with a representation that has not previously

Table 15. Pathway Curation task primary evaluation results by event type

\begin{tabular}{|c|c|c|c|c|c|c|}
\hline \multirow[t]{2}{*}{ Event } & \multicolumn{3}{|c|}{$\mathrm{NaCTeM}$} & \multicolumn{3}{|c|}{ TEES-2.1 } \\
\hline & recall & prec. & F-score & recall & prec. & F-score \\
\hline CONVERSION & 34.33 & 35.48 & 34.90 & 35.82 & 42.86 & 39.02 \\
\hline PHOSPHORYLATION & 62.46 & 55.94 & 59.02 & 53.40 & 66.00 & 59.03 \\
\hline DEPHOSPHORYLATION & 45.00 & 56.25 & 50.00 & 35.00 & 77.78 & 48.28 \\
\hline ACETYLATION & 69.57 & 72.73 & 71.11 & 82.61 & 76.00 & 79.17 \\
\hline DEACETYLATION & 33.33 & 33.33 & 33.33 & 0.00 & 0.00 & 0.00 \\
\hline METHYLATION & 42.86 & 60.00 & 50.00 & 57.14 & 80.00 & 66.67 \\
\hline DEMETHYLATION & 100.00 & 100.00 & 100.00 & 100.00 & 100.00 & 100.00 \\
\hline UBIQUITINATION & 52.94 & 64.29 & 58.06 & 58.82 & 76.92 & 66.67 \\
\hline DEUBIQUITINATION & 100.00 & 100.00 & 100.00 & 100.00 & 100.00 & 100.00 \\
\hline LOCALIZATION & 42.25 & 61.22 & 50.00 & 43.66 & 54.39 & 48.44 \\
\hline TRANSPORT & 65.52 & 61.29 & 63.33 & 56.55 & 59.85 & 58.16 \\
\hline GENE EXPRESSION & 90.65 & 83.15 & 86.74 & 84.55 & 79.39 & 81.89 \\
\hline TRANSCRIPTION & 71.15 & 82.22 & 76.29 & 57.69 & 73.17 & 64.52 \\
\hline TRANSLATION & 0.00 & 0.00 & 0.00 & 50.00 & 100.00 & 66.67 \\
\hline Simple-total & 66.42 & 64.80 & 65.60 & 60.40 & 67.87 & 63.92 \\
\hline DEGRADATION & 78.57 & 89.19 & 83.54 & 78.57 & 78.57 & 78.57 \\
\hline ACTIVATION & 78.54 & 70.96 & 74.56 & 72.06 & 72.06 & 72.06 \\
\hline INACTIVATION & 44.62 & 55.77 & 49.57 & 38.46 & 45.45 & 41.67 \\
\hline BINDING & 64.96 & 47.30 & 54.74 & 53.96 & 53.96 & 53.96 \\
\hline DISSOCIATION & 38.46 & 46.88 & 42.25 & 35.90 & 45.16 & 40.00 \\
\hline PATHWAY & 84.91 & 75.50 & 79.93 & 70.94 & 75.50 & 73.15 \\
\hline General-total & 69.07 & 62.69 & 65.72 & 61.16 & 65.74 & 63.37 \\
\hline REGULATION & 33.33 & 33.97 & 33.65 & 29.73 & 39.51 & 33.93 \\
\hline POSITIVE REGULATION & 35.49 & 42.81 & 38.81 & 34.51 & 45.45 & 39.23 \\
\hline NEGATIVE REGULATION & 45.75 & 50.64 & 48.07 & 41.02 & 47.37 & 43.97 \\
\hline Regulation-total & 37.73 & 42.79 & 40.10 & 35.17 & 44.76 & 39.39 \\
\hline Sub-total & 53.47 & 53.96 & 53.72 & 48.23 & 56.22 & 51.92 \\
\hline NEGATION & 24.52 & 35.87 & 29.13 & 25.16 & 41.30 & 31.27 \\
\hline SPECULATION & 15.79 & 22.22 & 18.46 & 0.00 & 0.00 & 0.00 \\
\hline Modification-total & 23.56 & 34.65 & 28.05 & 22.41 & 40.00 & 28.73 \\
\hline Total & 52.23 & 53.48 & 52.84 & 47.15 & 55.78 & 51.10 \\
\hline
\end{tabular}


been directly applied in event extraction task can be addressed with established technology without clear decreases in performance compared to established tasks [35].

\section{Additional evaluation results}

We next present evaluation results under different variants of the evaluation criteria. Note that the supplementary evaluation results presented in this section do not supersede the primary evaluation results (Tables 12 and 14), which remain the official results of the shared task.

Table 16 shows the CG task results for the core extraction targets. The F-score results show improvements ranging between $2.6-4.7 \%$ points for the various systems, reflecting the comparative simplicity of the core targets and the frequency of non-core targets: approximately $8 \%$ of test set events involve event modifications, i.e. NEGATION or SPECULATION annotations, and 6\% involve non-core arguments. The greatest benefit is observed for the NCBI system, which unlike the highest-ranking two systems does not attempt to predict event modifications.

Table 17 gives the core task results for the Pathway Curation task. The observed effect is more modest than for the CG task, likely reflecting both the lower frequency of non-core targets ( $4 \%$ of events have modifications, 6\% non-core arguments) and the fact that both participating systems predict modifications and all event arguments.

Table 18 summarizes the evaluation results for the CG task with the single partial penalty criterion. For the full task, this criterion provides for most systems comparable F-scores as evaluation on the core targets only (Table 16), although with a different precision/recall balance. Evaluation with the single partial penalty criterion

Table 16. Cancer Genetics task core evaluation results

\begin{tabular}{lllll}
\hline Team & recall & prec. & F-score & $\boldsymbol{\Delta}_{\boldsymbol{f}}$ \\
\hline TEES-2.1 & 52.14 & 66.18 & 58.33 & 2.92 \\
NaCTeM & 53.32 & 58.98 & 56.01 & 3.92 \\
NCBI & 43.33 & 62.07 & 51.04 & 4.66 \\
RelAgent & 44.82 & 52.40 & 48.32 & 3.00 \\
UET-NII & 22.08 & 65.21 & 33.00 & 3.06 \\
ISI & 18.57 & 49.93 & 27.08 & 2.61 \\
\hline
\end{tabular}

The $\Delta_{f}$ column shows absolute difference to the primary evaluation F-score.

Table 17. Pathway Curation task core evaluation results

\begin{tabular}{lllll}
\hline Team & recall & prec. & F-score & $\boldsymbol{\Delta}_{\boldsymbol{f}}$ \\
\hline NaCTeM & 54.14 & 54.78 & 54.46 & 1.62 \\
TEES-2.1 & 49.49 & 57.02 & 52.99 & 1.89 \\
\hline
\end{tabular}

The $\Delta_{f}$ column shows absolute difference to the primary evaluation F-score.
Table 18. Cancer Genetics task evaluation results with single partial penalty

\begin{tabular}{lllllllll}
\hline \multicolumn{4}{c}{ Primary (full task) } & \multicolumn{4}{c}{ Core } \\
\hline Team & recall & prec. & F-score & $\boldsymbol{\Delta}_{\boldsymbol{f}}$ & recall & prec. & F-score & $\boldsymbol{\Delta}_{\boldsymbol{f}}$ \\
\hline TEES-2.1 & 50.64 & 68.78 & 58.33 & 2.92 & 53.72 & 70.49 & 60.97 & 2.64 \\
NaCTeM & 50.70 & 61.88 & 55.74 & 3.65 & 55.03 & 64.43 & 59.36 & 3.35 \\
NCBI & 39.75 & 65.97 & 49.61 & 3.23 & 44.35 & 68.54 & 53.85 & 2.81 \\
RelAgent & 43.47 & 54.39 & 48.32 & 3.00 & 46.55 & 57.21 & 51.33 & 3.01 \\
UET-NII & 22.35 & 67.01 & 33.52 & 3.58 & 24.85 & 68.65 & 36.49 & 3.49 \\
ISI & 17.65 & 51.17 & 26.25 & 1.78 & 19.92 & 52.96 & 28.95 & 1.87 \\
\hline
\end{tabular}

The $\Delta_{f}$ columns show absolute difference to the corresponding F-scores without single partial penalty.

on the core targets shows that the relative improvements in F-score from these two variants of the primary evaluation are nearly additive, indicating that the variants address different aspects of the task.

The Pathway Curation task results with single partial penalty are shown in Table 19. For this task, the effect of this relaxed evaluation criterion is somewhat greater than that of considering core targets only, yet more limited than for the CG task. However, as for the CG task, the effects of the two variants are largely additive, with their combination providing a 4-5\% point increase over the primary F-score results. We note that the effect of the single partial penalty criterion for the CG and PC task results broadly parallels its effect on the results of the BioNLP ST'11 EPI and ID tasks for which it was originally proposed [36].

\section{Discussion}

We next summarize some of the main findings of the CG and PC task evaluations, consider specific challenges and possible ways of addressing these, and discuss the significance of the task results. Overall, we find that the best results in both the CG and PC tasks broadly parallel those achieved in similarly structured event extraction tasks in the BioNLP ST'09 [28] and ' $11[35,36]$. This suggests that the difficulty of the extraction task is not primarily determined by the factors that have varied between these tasks, such as the domain of the source texts (cancer, pathways, infectious diseases, etc.), the level of biological organization (molecular, cellular, tissue, etc.), the ontological basis applied in the annotation

Table 19. Pathway Curation task evaluation results with single partial penalty

\begin{tabular}{lllllllll}
\hline \multicolumn{4}{c}{ Primary (full task) } & \multicolumn{4}{c}{ Core $\boldsymbol{\Delta}_{\boldsymbol{f}}$} \\
\hline Team & recall & prec. & F-score & $\boldsymbol{\Delta}_{\boldsymbol{f}}$ & recall & prec. & F-score & $\boldsymbol{\Delta}_{\boldsymbol{f}}$ \\
\hline NaCTeM & 54.14 & 57.02 & 55.54 & 2.70 & 56.12 & 58.34 & 57.21 & 2.75 \\
TEES-2.1 & 49.66 & 58.77 & 53.83 & 2.73 & 52.13 & 59.85 & 55.72 & 2.73 \\
\hline
\end{tabular}

The $\Delta_{f}$ columns show absolute difference to the corresponding F-scores without single partial penalty. 
(GO, SBO), or, remarkably, even the number of different entity or event types targeted in the task. The detailed results bear out this observation (Tables 13 and 15). For example, we find that extraction performance for events taking only single arguments is comparatively high across the different categories outlined above (Figure 6).

Similarly, complex extraction targets such as events involving multiple arguments, events that recursively involve another event, and event modifications that often involve non-local "triggers" that are not explicitly annotated (Figure 7) represent challenges in all task settings. The relative difficulty of these extraction targets is well established, and much of it is inherent: it is more challenging to extract a structure involving three elements than one involving two, and the primary evaluation metrics of the shared tasks make no attempt to normalize away such effects. Nevertheless, the finding that most errors are found in these types of structures suggests that substantial advances in extraction performance may require extraction approaches emphasizing global features and joint models rather than the comparatively local approaches that currently dominate biomedical event extraction.

Previous detailed analyses of system errors [28,36] identified a number of specific properties that further characterize hard-to-extract events, including complex syntactic structure, participants identified through coreference, or reference to entities via relations such as part-of. Despite supporting tasks organized to address some of these challenges as part of the BioNLP ST'11 $[85,86]$ and some successful implementations in event extraction systems [84], no system participating in the current tasks attempted integration of coreference resolution or entity relation extraction with event extraction. The combination of such analysis components is technically challenging, but it is likely that many events cannot be reliably extracted without such comprehensive integration.

It must also be noted that measured extraction performance is limited by the quality and consistency of the gold standard annotation: even domain experts trained to perform event annotation may reach no more than $60 \%$

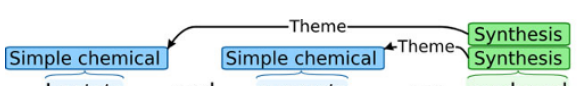

Lactate and are pyruvate

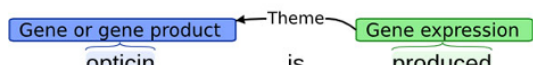

opticin

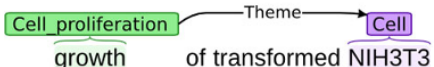

growth of transformed $\overparen{\mathrm{NIH} 3 \mathrm{~T} 3}$

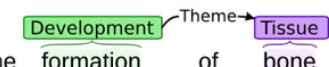

Growth $\Upsilon^{\text {Theme- }}$ Cancer

growth of adenocarcinoma

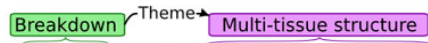

damage of the peritoneal membrane

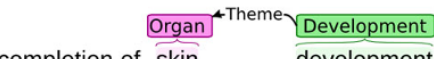

development

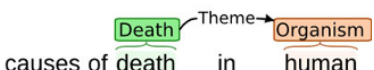

Figure 6 Simple events. Events with single arguments are reliably extracted regardless of factors such as text domain or level or biological organization.

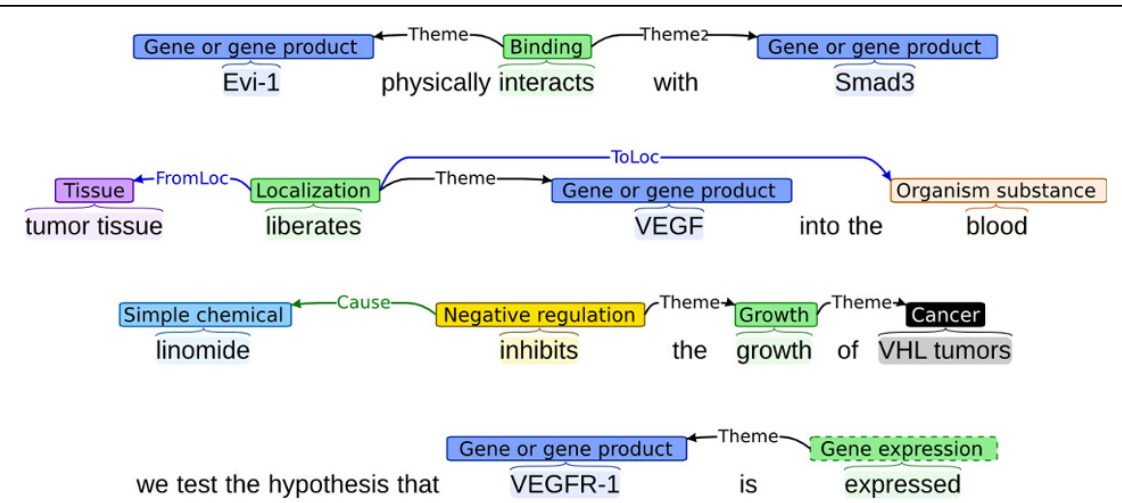

Figure 7 Complex events. Events involving multiple participants, recursive structure, and modifications continue to represent challenges for extraction. 
F-score in cases, and 80\% F-score remains a difficult target even for highly trained human annotators. The evaluation criteria can also be questioned: results using different criteria showed relative reductions in F-score error of $10 \%$ or more for most systems (Tables 18 and 19). These differences raise a question of which result is "correct", or which reflects the "true" performance of the systems. Naturally, no single number can adequately represent system performance in full, and preferences between different evaluation criteria are taskdependent and somewhat subjective. However, we note that the results of previous manual evaluations of system outputs (e.g. [36,87]) suggest that users may perceive the quality of system outputs as substantially higher than the primary evaluation results indicate, lending support to the application of also more relaxed criteria. Finally, we note that although evaluation variants produce substantial effects on absolute F-scores, all criteria agree on which of any two systems performs better. Thus, the primary results are stable in their ranking of systems by performance in both the CG and PC tasks.

\section{Conclusions}

We have presented the Cancer Genetics and Pathway Curation tasks, two event extraction tasks that were newly introduced in the BioNLP Shared Task 2013. Both tasks are motivated by the needs of maintaining comprehensive and up-to-date information in the face of the enormous size and rapid growth of the biomedical domain scientific literature. For the CG task, such information can be used to develop knowledge bases on the molecular mechanisms underlying cancer; for the PC task, for developing, evaluating and maintaining molecular pathway models using representations such as SBML and BioPAX.

The two tasks both involve a number of aspects not previously considered in BioNLP ST settings. For the CG task, the most notable novel points are that the task addresses entities and events at all levels of biological organization from the molecular to the whole organism and involves pathological as well as physiological processes. The PC task stands out in particular in defining the structure of its extraction targets explicitly with reference to major pathway model representations and their types on the basis of the Systems Biology Ontology, thus aligning the extraction task closely with the needs of pathway curation efforts. Each of the tasks introduces a new, manually annotated corpus substantially extending on previously available resources. Both corpora draw their texts from PubMed abstracts, with the CG corpus containing annotations of over 17,000 events in 600 documents and the PC corpus over 12,000 events in 525 documents.

Six groups participated in the CG task, applying a broad range of extraction approaches including machine learning-based pipelines, a joint pattern matching-based approach, a rule-based approach and two parsing-based approaches. Two groups participated in the PC task, both applying well-established pipeline systems using support vector machines. All participating systems applied detailed analysis of sentence structure, most commonly in the form of full dependency parses. The best result achieved in the CG task was an F-score of $55.4 \%$ by the TEES-2.1 system, and the best result in the PC task was an F-score of $52.8 \%$ by the EventMine system. Both of the top-ranking systems are machine learning-based pipeline systems that have achieved state-ofthe-art results in many previously proposed event extraction tasks.

The performance of the top-ranking systems at the two tasks is broadly in the range of the best results achieved at similar previously proposed event extraction tasks, indicating that current event extraction methods generalize well to meet the novel challenges represented by the CG and PC tasks.

Following the convention established in the first BioNLP Shared Task, both the Cancer Genetics and Pathway Curation tasks will continue as open challenges available to all interested participants. The corpora, evaluation tools, and supporting resources are available under open licenses from the BioNLP Shared Task homepage http://2013.bionlp-st.org/.

\section{Competing interests}

The authors declare that they have no competing interests.

\section{Authors' contributions}

SP and TO conceived of the tasks. SP coordinated the CG task organization, drafted the manuscript, and implemented evaluation and support tools. TO coordinated the PC task organization and led the CG and PC annotation efforts. RR and AR contributed to preparation of tools for the PC task. HC, SJ and SC contributed to the design and manual annotation of the PC task. SA participated in the design and coordination of both tasks and JT participated in the coordination of the overall shared task.

\section{Acknowledgements}

We wish to thank the BioNLP ST 2013 participants and supporting resource providers for their invaluable contributions to making these tasks a success. We would like to thank Yonghwa Jo and Hyeyeon Choi for their

contribution to the development of the Pathway Curation task document relevance judgment annotation criteria. We also wish to thank Hyun Uk Kim, Jinki Kim and Kyusang Hwang of KAIST for their efforts in producing the Pathway Curation task annotation. The Pathway Curation task organization was a part of joint research of KISTI and NaCTeM. This work was partially supported by the Biotechnology and Biological Sciences Research Council (BBSRC) [BB/G53025X/1]. Costs for publication were funded by the University of Turku.

This article has been published as part of BMC Bioinformatics Volume 16 Supplement 10, 2015: BioNLP Shared Task 2013: Part 1. The full contents of the supplement are available online at http://www.biomedcentral.com/ bmcbioinformatics/supplements/16/S10.

\section{Authors' details}

${ }^{1}$ Department of Information technology, University of Turku, Turku, Finland. 2textimi, Tokyo, Japan. ${ }^{3}$ National Centre for Text Mining and School of Computer Science, University of Manchester, Manchester, UK. ${ }^{4}$ Software 
Research Center, Korea Institute of Science and Technology Information (KISTI), Daejeon, South Korea. ${ }^{5}$ Department of Applied Information Science, University of Science and Technology (UST), Daejeon, South Korea.

${ }^{6}$ Department of Library and Information Science, Kyonggi University, Suwon, South Korea. ${ }^{7}$ Microsoft Research Asia, Beijing, China.

Published: 13 July 2015

\section{References}

1. Nédellec C, Bossy R, Kim JD, Kim JJ, Ohta T, Pyysalo S, Zweigenbaum P. Overview of BioNLP Shared Task 2013. Proceedings of the BioNLP Shared Task 2013 Workshop 2013, 1-7.

2. Bossy R, Golik W, Ratkovic Z, Valsamou D, Bessières P, Nédellec C: An Overview of the Gene Regulation Network and the Bacteria Biotope Tasks in BioNLP'13 Shared Task. BMC Bioinformatics 2014, 16(Suppl 10).

3. Kim JD, Kim JJ, Han X, Rebholz-Schuhmann D: Extending the evaluation of Genia Event task toward knowledge base construction and comparison to Gene Regulation Ontology task. BMC Bioinformatics 2014.

4. Pyysalo S, Ohta T, Ananiadou S: Overview of the Cancer Genetics (CG) task of BioNLP Shared Task 2013. Proceedings of the BioNLP Shared Task 2013 Workshop 2013, 58-66.

5. Ohta T, Pyysalo S, Rak R, Rowley A, Chun HW, Jung SJ, et al: Overview of the pathway curation (PC) task of BioNLP Shared Task 2013. Proceedings of the BioNLP Shared Task 2013 Workshop 2013, 67-75.

6. Hanahan D, Weinberg RA: The hallmarks of cancer. Cell 2000, 100(1):57-70.

7. Haber DA, Gray NS, Baselga J: The evolving war on cancer. Cell 2011, 145(1):19-24.

8. PubMed. [http://www.pubmed.gov/].

9. Hucka M, Finney A, Sauro HM, Bolouri H, Doyle JC, Kitano H, et al: The systems biology markup language (SBML): a medium for representation and exchange of biochemical network models. Bioinformatics 2003, 19(4):524-531.

10. Systems Biology Markup Language (SBML). [http://sbml.org/].

11. Demir E, Cary MP, Paley S, Fukuda K, Lemer C, Vastrik I, et al: The BioPAX community standard for pathway data sharing. Nature Biotechnology 2010, 28(9):935-942.

12. BioPAX. [http://www.biopax.org/].

13. Kitano H: Systems biology: a brief overview. Science 2002, 295(5560):1662-1664.

14. Li C, Donizelli M, Rodriguez N, Dharuri H, Endler L, Chelliah V, et al: BioModels Database: An enhanced, curated and annotated resource for published quantitative kinetic models. BMC Systems Biology 2010, 4:92.

15. Mi H, Thomas P: PANTHER pathway: an ontology-based pathway database coupled with data analysis tools. Methods Mol Biol 2009, 563:123-140

16. Yeh A, Morgan A, Colosimo M, Hirschman L: BioCreAtlvE Task 1A: gene mention finding evaluation. BMC Bioinformatics 2005, 6(Suppl 1):S2.

17. Smith L, Tanabe L, Ando R, Kuo CJ, Chung IF, Hsu CN, et al: Overview of BioCreative II gene mention recognition. Genome Biology 2008, 9(Suppl 2):S2.

18. Krallinger M, Leitner F, Valencia A: Assessment of the Second BioCreative PPI task: Automatic Extraction of Protein-Protein Interactions. Proceedings of Second BioCreative Challenge Evaluation Workshop 2007, 29-39.

19. Pyysalo S, Airola A, Heimonen J, Björne J, Ginter F, Salakoski T: Comparative analysis of five protein-protein interaction corpora. BMC Bioinformatics 2008, 9(Suppl 3):S6.

20. Tikk D, Thomas P, Palaga P, Hakenberg J, Leser U: A Comprehensive Benchmark of Kernel Methods to Extract Protein-Protein Interactions from Literature. PLoS Comput Biol 2010, 6(7):e1000837.

21. Kim JD, Ohta T, Pyysalo S, Kano Y, Tsujii J: Overview of BioNLP'09 Shared Task on Event Extraction. BioNLP '09 Proceedings of the Workshop on Current Trends in Biomedical Natural Language Processing: Shared Task 2009, $1-9$.

22. Kim JD, Pyysalo S, Ohta T, Bossy R, Tsujii J: Overview of BioNLP Shared Task 2011. Proceedings of BioNLP Shared Task 2011 Workshop 2011, 1-6.

23. Oda K, Kim JD, Ohta T, Okanohara D, Matsuzaki T, Tateisi $Y$, Tsujii J: New challenges for text mining: mapping between text and manually curated pathways. BMC Bioinformatics 2008, 9(Suppl 3):S5.

24. Ohta T, Pyysalo S, Tsujii J: From pathways to biomolecular events: opportunities and challenges. Proceedings of the 2011 Workshop on Biomedical Natural Language Processing 2011, 105-113.
25. Courtot $M$, Juty $N$, Knüpfer C, Waltemath $D$, Zhukova A, Dräger A, et al: Controlled vocabularies and semantics in systems biology. Molecular Systems Biology 2011, 7:543.

26. SBO::Systems Biology Ontology. [http://www.ebi.ac.uk/sbo/]

27. Ananiadou S, Pyysalo S, Tsujii J, Kell DB: Event extraction for systems biology by text mining the literature. Trends in Biotechnology 2010, 28(7):381-390

28. Kim JD, Ohta T, Pyysalo S, Kano Y, Tsujii J: Extracting Bio-molecular Events from Literature - the BioNLP'09 Shared Task. Computational Intelligence 2011, 27(4):513-540.

29. Nadeau D, Sekine S: A survey of named entity recognition and classification. Lingvisticae Investigationes 2007, 30:3-26.

30. Ananiadou S, McNaught J: Text Mining for Biology and Biomedicine Citeseer; 2006.

31. Krallinger $M$, Leitner $F$, Rabal $O$, Vazquez M, Oyarzabal J, Valencia A: Overview of the chemical compound and drug name recognition (CHEMDNER) task. BioCreative Challenge Evaluation Workshop 2013, 2:2-33.

32. Vincze V, Szarvas G, Farkas R, Móra G, Csirik J: The BioScope corpus: biomedical texts annotated for uncertainty, negation and their scopes. BMC Bioinformatics 2008, 9(Suppl 11):S9.

33. Farkas R, Vincze V, Móra G, Csirik J, Szarvas G: The CoNLL-2010 shared task: learning to detect hedges and their scope in natural language text. PCONLL '10: Shared Task Proceedings of the Fourteenth Conference on Computational Natural Language Learning - Shared Task 2010, 1-12.

34. BioNLP Shared Task 2013 File Formats. [http://2013.bionlp-st.org/fileformats].

35. Kim JD, Nguyen N, Wang Y, Tsujii J, Takagi T, Yonezawa A: The Genia Event and Protein Coreference tasks of the BioNLP Shared Task 2011. BMC Bioinformatics 2012, 13(Suppl 11):S1.

36. Pyysalo S, Ohta T, Rak R, Sullivan D, Mao C, Wang C, et al: Overview of the ID, EPI and REL tasks of BioNLP Shared Task 2011. BMC Bioinformatics 2012, 13(Suppl 11):S2.

37. Haendel MA, Neuhaus F, Osumi-Sutherland D, Mabee PM, Mejino JL Jr, Mungall CJ, Smith B: CARO - The Common Anatomy Reference Ontology. Anatomy Ontologies for Bioinformatics 2008, 6:327-349.

38. Rosse C, Mejino JL Jr: A reference ontology for biomedical informatics: the Foundational Model of Anatomy. Journal of Biomedical Informatics 2003, 36(6):478-500.

39. Ohta T, Pyysalo S, Tsujii J, Ananiadou S: Open-domain anatomical entity mention detection. $A C L$ ' 12 Proceedings of the Workshop on Detecting Structure in Scholarly Discourse 2012, 27-36.

40. Maglott D, Ostell J, Pruitt KD, Tatusova T: Entrez Gene: gene-centered information at NCBI. Nucleic Acids Research 2005, 33(Database issue):D54-D58.

41. UniProt Consortium: Ongoing and future developments at the Universal Protein Resource. Nucleic Acids Research 2011, 39(Database issue): D214-D219.

42. Finn RD, Mistry J, Tate J, Coggill P, Heger A, Pollington JE, Gavin OL, Gunasekaran P, et al: The Pfam protein families database. Nucleic Acids Research 2010, 38(Database issue):D211-D222.

43. Greene LH, Lewis TE, Addou S, Cuff A, Dallman T, Dibley M, et al: The CATH domain structure database: new protocols and classification levels give a more comprehensive resource for exploring evolution. Nucleic Acids Research 2007, 35(Database issue):D291-D297.

44. Degtyarenko K, De Matos P, Ennis M, Hastings J, Zbinden M, Mcnaught A, et al: ChEBI: a database and ontology for chemical entities of biological interest. Nucleic Acids Research 2008, 36(Database issue):D344-D350.

45. Mungall CJ, Torniai C, Gkoutos GV, Lewis SE, Haendel MA, et al: Uberon, an integrative multi-species anatomy ontology. Genome Biol 2012, 13(1):R5.

46. Bard J, Rhee SY, Ashburner M: An ontology for cell types. Genome Biol 2005, 6(2):R21.

47. Federhen S: The NCBI taxonomy database. Nucleic Acids Research 2012 40(Database issue):D136-D143.

48. Ashburner M, Ball CA, Blake JA, Botstein D, Butler H, Cherry JM, et al: Gene ontology: tool for the unification of biology. The Gene Ontology Consortium. Nature Genetics 2000, 25(1):25-29.

49. Brinkman RR, Courtot M, Derom D, Fostel JM, He Y, Lord P, et al: Modeling biomedical experimental processes with OBI. J Biomed Semantics 2010, , Suppl 1: S7.

50. Pyysalo S, Ohta T, Miwa M, Cho HC, Tsujii J, Ananiadou S: Event extraction across multiple levels of biological organization. Bioinformatics 2012, 28(18):i575-i581. 
51. Corbett P, Copestake A: Cascaded classifiers for confidence-based chemical named entity recognition. BMC Bioinformatics 2008, 9(Suppl 11):S4.

52. Leaman R, Gonzalez G: BANNER: an executable survey of advances in biomedical named entity recognition. Pac Symp Biocomput 2008, 652-663.

53. Cho HC: NERsuite: A Named Entity Recognition toolkit 2010 [http://nersuite. nlplab.org/.

54. Wilbur J, Smith L, Tanabe L: BioCreative 2. Gene Mention Task. In Proceedings of Second BioCreative Challenge Evaluation Workshop Hirschman L, Krallinger M, Valencia A 2007, 7-16.

55. Pyysalo $S$, Ananiadou S: Anatomical entity mention recognition at literature scale. Bioinformatics 2013, 30(6):868-875.

56. Gerner M, Nenadic G, Bergman CM: LINNAEUS: a species name identification system for biomedical literature. BMC Bioinformatics 2010, 11:85.

57. Stenetorp P, Topić G, Pyysalo S, Ohta T, Kim JD, Tsujii J: BioNLP Shared Task 2011: Supporting Resources. Proceedings of BioNLP Shared Task 2011 Workshop 2011, 112-120

58. Rak R, Rowley A, Black W, Ananiadou S: Argo: an integrative, interactive, text mining-based workbench supporting curation. Database (Oxford) 2012, 2012:bas010.

59. Stenetorp P, Pyysalo S, Topić G, Ohta T, Ananiadou S, Tsujii J: BRAT: a webbased tool for NLP-assisted text annotation. EACL'12 Proceedings of the Demonstrations at the 13th Conference of the European Chapter of the Association for Computational Linguistics 2012, 102-107.

60. Kemper B, Matsuzaki T, Matsuoka Y, Tsuruoka Y, Kitano H, Ananiadou S, Tsujii J: PathText: a text mining integrator for biological pathway visualizations. Bioinformatics 2010, 26(12):i374-i381.

61. Miwa M, Ohta T, Rak R, Rowley A, Kell DB, Pyysalo S, Ananiadou S: A method for integrating and ranking the evidence for biochemical pathways by mining reactions from text. Bioinformatics 2013, 29(13):i44-i52.

62. Noreen EW: Computer-Intensive Methods for Testing Hypotheses: An Introduction Wiley; 1989.

63. Chinchor N: The statistical significance of the MUC-4 results. MUC4 ' 92 Proceedings of the 4th conference on Message understanding 1992, 30-50.

64. Ohta T, Pyysalo S, Tsujii J: Overview of the Epigenetics and Posttranslational Modifications (EPI) task of BioNLP Shared Task 2011. Proceedings of BioNLP Shared Task 2011 Workshop 2011, 16-25.

65. Liu H, Christiansen T, Baumgartner WA Jr, Verspoor K, et al: BioLemmatizer: a lemmatization tool for morphological processing of biomedical text. J Biomed Semantics 2012, 3(3).

66. McClosky D: Any Domain Parsing: Automatic Domain Adaptation for Natural Language Parsing. PhD thesis Department of Computer Science, Brown University; 2009.

67. Charniak E, Johnson M: Coarse-to-Fine $n$-Best Parsing and MaxEnt Discriminative Reranking. ACL '05 Proceedings of the 43rd Annual Meeting on Association for Computational Linguistics5 2005, 173-180.

68. de Marneffe MC, MacCartney B, Manning CD: Generating typed dependency parses from phrase structure parses. Proceedings of LREC 2006, 6:449-454.

69. Miyao Y, Tsujii J: Feature forest models for probabilistic HPSG parsing. Computational Linguistics 2008, 34(1):35-80.

70. Sagae K, Tsujii J: Dependency parsing and domain adaptation with LR models and parser ensembles. Proceedings of the CoNLL Shared Task Session of EMNLP-CoNLL 2007 2007, 1044-1050.

71. Ramanan S, Nathan PS: Performance and limitations of the linguistically motivated Cocoa/Peaberry system in a broad biological domain. Proceedings of the BioNLP Shared Task 2013 Workshop 2013, 86-93.

72. Miwa M, Pyysalo S, Ohta T, Ananiadou S: Wide coverage biomedical event extraction using multiple partially overlapping corpora. $B M C$ Bioinformatics 2013, 14:175.

73. Thompson P, Nawaz R, McNaught J, Ananiadou S: Enriching a biomedical event corpus with meta-knowledge annotation. BMC Bioinformatics 2011, 12:393.

74. Pyysalo S, Ohta T, Miwa M, Tsujii J: Towards Exhaustive Protein Modification Event Extraction. Proceedings of the 2011 Workshop on Biomedical Natural Language Processing 2011, 114-123.

75. Ohta T, Pyysalo S, Miwa M, Tsujii J: Event extraction for DNA methylation. Journal of Biomedical Semantics 2011, 2(Suppl 5):S2.

76. Björne J, Heimonen J, Ginter F, Airola A, Pahikkala T, Salakoski T: Extracting Contextualized Complex Biological Events with Rich Graph-Based Feature Sets. Computational Intelligence 2011, 27(4):541-557.

77. Björne J: TEES: Turku Event Extraction System. 2012 [http://jbjorne.github. io/TEES/].
78. Miwa M, Thompson P, McNaught J, Kell D, Ananiadou S: Extracting semantically enriched events from biomedical literature. $B M C$ Bioinformatics 2012, 13:108.

79. Miwa M: EventMine: Event Extraction System for Biomedical Text. 2013 [http://nactem.ac.uk/EventMine/].

80. Liu H, Komandur R, Verspoor K: From graphs to events: A subgraph matching approach for information extraction from biomedical text. Proceedings of the 2011 Workshop on Biomedical Natural Language Processing 2011, 164-172.

81. Liu H, Hunter L, Kešelj V, Verspoor K: Approximate Subgraph MatchingBased Literature Mining for Biomedical Events and Relations. PLoS One 2013, 8(4):e60954.

82. Björne J, Heimonen J, Ginter F, Airola A, Pahikkala T, Salakoski T: Extracting Complex Biological Events with Rich Graph-Based Feature Sets. BioNLP '09 Proceedings of the Workshop on Current Trends in Biomedical Natural Language Processing: Shared Task 2009, 10-18.

83. Björne J, Salakoski T: Generalizing biomedical event extraction. Proceedings of the 2011 Workshop on Biomedical Natural Language Processing 2011, 183-191.

84. Miwa M, Thompson P, Ananiadou S: Boosting automatic event extraction from the literature using domain adaptation and coreference resolution. Bioinformatics 2012, 28(13):1759-1765.

85. Nguyen N, Kim JD, Tsujii J: Overview of the Protein Coreference task in BioNLP Shared Task 2011. Proceedings of BioNLP Shared Task 2011 Workshop 2011, 74-82.

86. Pyysalo S, Ohta T, Tsujii J: Overview of the Entity Relations (REL) supporting task of BioNLP Shared Task 2011. Proceedings of BioNLP Shared Task 2011 Workshop 2011, 83-88.

87. Björne J, Ginter F, Pyysalo S, Tsujii J, Salakoski T: Complex event extraction at PubMed scale. Bioinformatics 2010, 26(12):i382-i390.

88. Caron E, Ghosh S, Matsuoka Y, Ashton-Beaucage D, Therrien M, Lemieux S, et al: A comprehensive map of the mTOR signaling network. Molecular Systems Biology 2010, 6:453

89. Oda K, Kitano H: A comprehensive map of the toll-like receptor signaling network. Molecular Systems Biology 2006, 2:2006.0015.

90. Kaizu K, Ghosh S, Matsuoka Y, Moriya H, Shimizu-Yoshida Y, Kitano H: A comprehensive molecular interaction map of the budding yeast cell cycle. Molecular Systems Biology 2010, 6:415.

91. Calzone L, Gelay A, Zinovyev A, Radvanyl F, Barillot E: A comprehensive modular map of molecular interactions in RB/E2F pathway. Molecular Systems Biology 2008, 4:173.

92. Oda K, Matsuoka Y, Funahashi A, Kitano H: A comprehensive pathway map of epidermal growth factor receptor signaling. Molecular Systems Biology 2005, 1:2005.0010

93. Duarte NC, Becker SA, Jamshidi N, Thiele I, Mo ML, Vo TD, et al: Global reconstruction of the human metabolic network based on genomic and bibliomic data. Proceedings of the National Academy of Sciences 2007, 104(6):1777-1782.

94. Björne J, Salakoski T: TEES 2.1: Automated Annotation Scheme Learning in the BioNLP 2013 Shared Task. Proceedings of the BioNLP Shared Task 2013 Workshop 2013, 16-25.

95. Björne J, Salakoski T: TEES 2.2: Biomedical Event Extraction for Diverse Corpora. BMC Bioinformatics 2014.

96. Miwa M, Ananiadou S: NaCTeM EventMine for BioNLP 2013 CG and PC tasks. Proceedings of the BioNLP Shared Task 2013 Workshop 2013, 94-98.

97. Miwa M, Ananiadou S: Adaptable, High Recall, Event Extraction System with Minimal Configuration. BMC Bioinformatics 2014.

98. Liu H, Verspoor K, Comeau D, MacKinlay A, Wilbur WJ: Generalizing an Approximate Subgraph Matching-based System to Extract Events in Molecular Biology and Cancer Genetics. Proceedings of the BioNLP Shared Task 2013 Workshop 2013, 76-85.

99. Liu H, Verspoor K, Comeau DC, MacKinlay A, Wilbur WJ: Optimizing graphbased patterns to extract biomedical events from the literature. BMC Bioinformatics 2014.

100. Tran MV, Collier N, Le HQ, Phi VT, Pham TB: Adapting a probabilistic Earley Parser for event decomposition in biomedical texts. Proceedings of the BioNLP Shared Task 2013 Workshop 2013.

doi:10.1186/1471-2105-16-S10-S2

Cite this article as: Pyysalo et al:: Overview of the Cancer Genetics and Pathway Curation tasks of BioNLP Shared Task 2013. BMC Bioinformatics 2015 16(Suppl 10):S2. 